\title{
Beitrag zur Anatomie, Physiologie und Pathologie der Portio vaginalis uteri. $\left.{ }^{1}\right)$
}

\author{
Von \\ Dr. Alfred Dührssen, \\ Assistent der geburtshülflichen Klinik der Königl. Charité und Privatdocent an der \\ Universität zu Berlin.
}

(Mït 15 Holzsehnitten und 6 Abbildungen anf Taf. XI u. XII.)

Von der Zeit an, wo ich begann, gynäkologische Operationscurse.am Schultze-Winckel'schen Phantome abzuhalten, fiel mir die eigenthümliche Gestaltsveränderung auf, welche die Wundflächen nach der keilförmigen Amputation der Portio erleiden. Selbst bei tiefer, unter dem Niveau der Scheidengewölbe erfolgter Absetzung sieht man nämlich, dass die entstandenen Wundfächen der vorderen und hinteren Lippe auf das vordere bezw. hintere Scheidengewölbe übergreifen. Nach der Veruähung sieht man ferner, dass die vernähten Seitenschnitte im seitlichen Scheidengewölbe liegen.

Bei Operationen an der Lebenden kann man ganz dieselbe Beobachtung machen. Der Grund, dass ich sie zuerst an der Leiche machte, lag einfach in den Schwierigkeiten, welche sich der Ausführung der vaginalen Exstirpation des Uterus an Präparaten entgegenstellten, bei denen schon die Portioamputation gemacht worden war. An diesen lagen die vernähten Wunden im Scheidengewölbe da, wo eigentlich der die Portio umkreisende Schnitt geführt werden sollte.

Wenn ich also bei der Ergründung dieser eigenthümlichen Retractionserscheinungen gewisse neue Thatsachen gefunden habe - und dass dem so ist, hoffe ich in dieser Arbeit darzulegen -, so verdanke ich die Anregung dazu den bei Operationscursen ge-

1) Die Resultate der vorliegenden Arbeit sind zum grössten Theile bereits auf dem internationalen Congresse zu Berlin und dem Gynäkologencongresse in Bonn mitgetheilt worden. 
这

$$
2 \text { bits }
$$
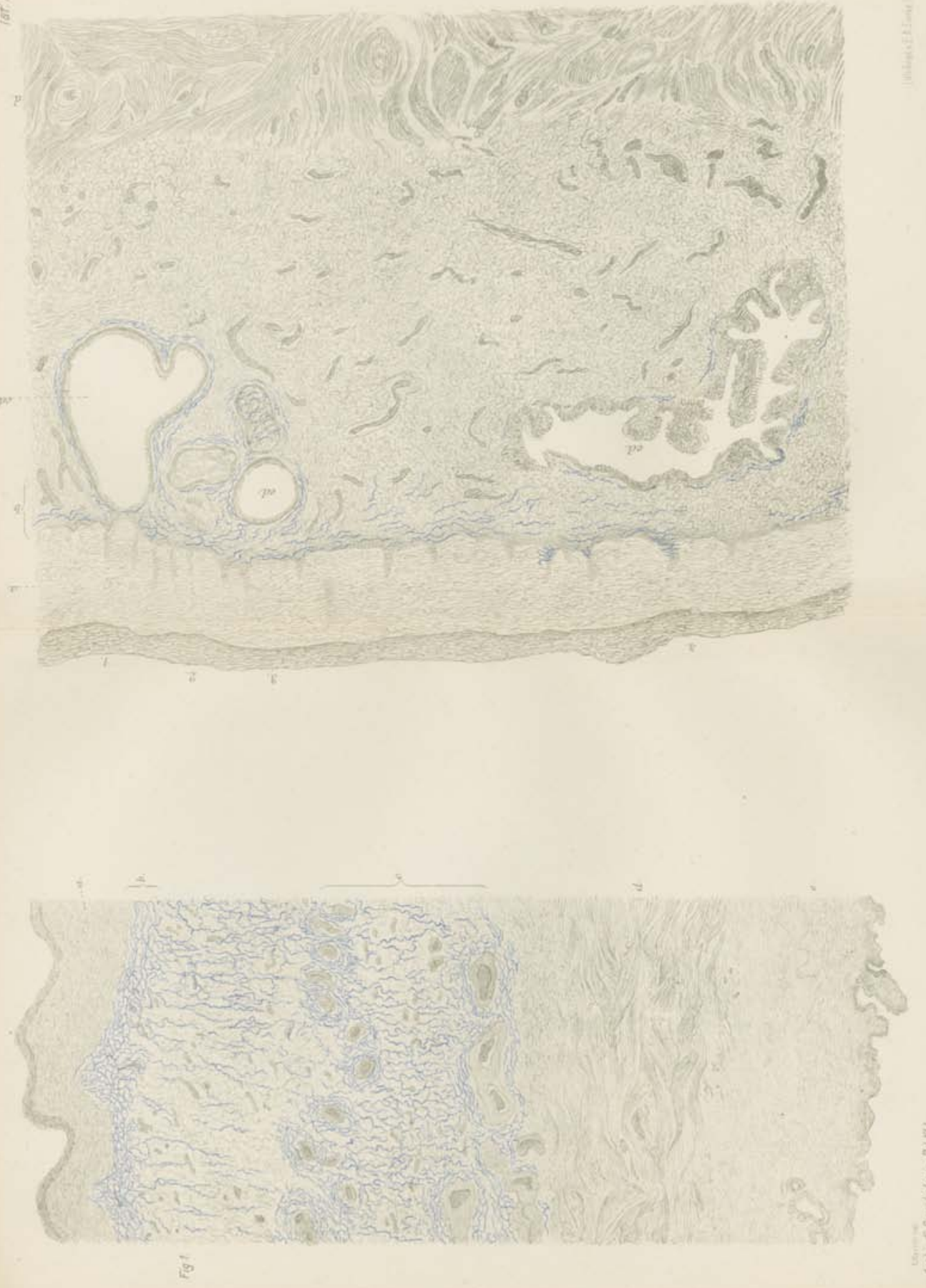


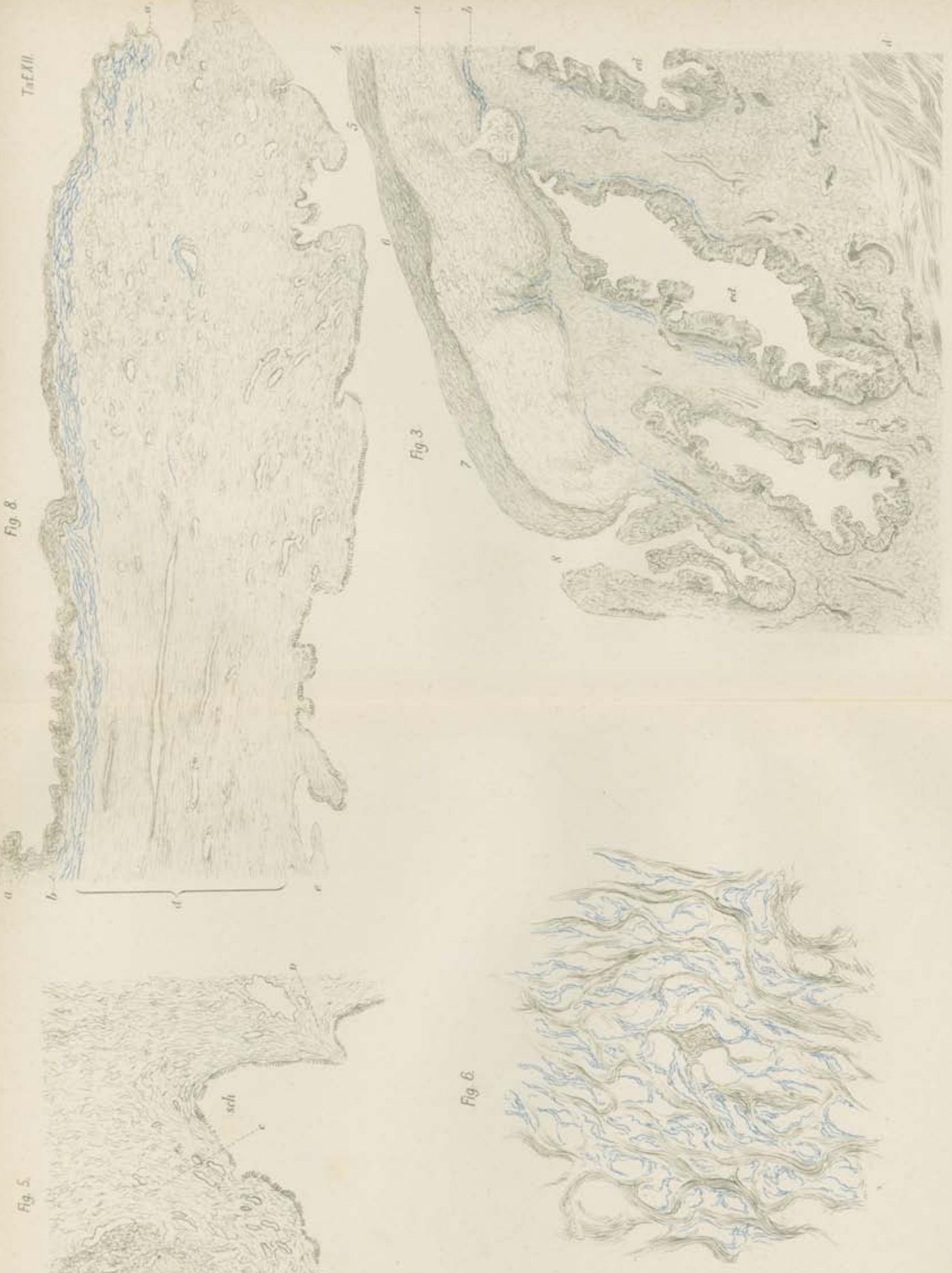


machten Beobachtungen. Es ist dies gerade ein Punkt, den Winckel') sehr mit Recht für den Werth dieser Uebungen ins Feld geführt hat.

Dass nach der Portioamputation - mag sie auch nur ein $1 \mathrm{~cm}$ hohes Stück der Portio wegnehmen - ungefähr die Hälfte der gesetzten Wunde im Scheidengewölbe liegt, davon kann man sich gerade auch an der Leiche in sehr bequemer Weise überzeugen. Führt man nämlich nach vollzogener Amputation den Finger in die Plica vesico-uterina oder in den Douglas'schen Raum, so stülpt der Finger an der vorderen Lippe den vorderen Theil der Wunde, an der hinteren Lippe den hinteren Theil nach abwärts. Es liegt also über der Wunde nicht etwa compacte Uterussubstanz, sondern in den genannten Abschnitten lockeres Gewebe und dicht darüber Peritoneum. Daher sieht man denn auch an den Präparaten sehr häufig, dass die Fäden, welche die Wunde an der hinteren Lippe vereinigten, im Douglas'schen Raume liegen.

Nachdem diese Beobachtungen an Hunderten von Uteri mein Interesse erregt hatten, unterwarf ich 50 Uteri einer genaueren, theils makroskopischen, theils mikroskopischen Untersuchung. Auf Sagittalschnitten durch den mit der Vagina im Zusammenhange befindlichen Uterus sieht man nun, wie nicht nur die Schleimhaut der Vaginalwand, sondern auch die tieferen Schichten derselben auf die Cervix übergehen. Und zwar geschieht dieser Uebergang in zwei Bündeln, einem oberen und einem unteren. Das obere breitet sich fächerförmig aus, geht aber nicht tief in die Substanz des Uterus hinein, das untere dagegen erstreckt sich bis nahezu zum Niveau des Muttermundes herab und schwillt zu einer solchen Stärke an, dass es die äussere Hälfte der Portio vaginalis einnimmt. Von diesem Verhalten kann man sich mit Leichtigkeit überzeugen, wenn man bei einem solchen Sagittalschnitte durch Uterus und Scheide an der Vaginalwand einen Zug ausübt. Es spannt sich dann die eben beschriebene Partie, also speciell an der Portio, die äussere Hälfte stärker an - eine Erscheinung, die unmöglich wäre, wenn die Vaginalwand sich nur einfach dem Uteruskörper anlegte, wenn sie nicht die eben beschriebene Fortsetzung in den Uterus hinein aufwiese.

Weitere Unterschiede zwischen der äusseren Hälfte der Portio und der inneren, die den Cervicalkanal umgiebt, sind bedingt

1) Lehrbuch der Frauenkrankheiten. 1886. Einleitung. 
durch den grösseren Gefässreichthum und die weiche Consistenz der äusseren Partie der Vaginalportion. Bei der mikroskopischen Betrachtung zeigt es sich, dass die äussere Partie in Wesentlichen aus Bindegewebe besteht, während die innere derbe Partie reich an glatter Muskulatur ist, deren Bündel vorwiegend longitudinal verlaufen.

Es waren dies die anatomischen Grundlagen, deren Bedeutung für die Entstehung der Para- und Perimetritis nach der Portioamputation ich in meinem auf dem internationalen Congresse zu Berlin gehaltenen Vortrage: „Ueber Operationen an der Portio" hervorhob.

In weiterer Verfolgung dieser Frage ergab sich mir eine gewisse Schwierigkeit in der Deutung des mikroskopischen Befundes in seiner Beziehung zu der so verschiedenen Consistenz der äusseren und inneren Partie der Portio. Ich sagte mir, dass ein Myom um so weicher sei, je mehr Muskelfasern es enthalte, um so härter, je reicher es an Bindegewebe sei. Wie kam es denn, dass sich dies an der Portio gerade umgekehrt verhielt, dass der an Muskelfasern reiche Theil eine feste, der äussere, mehr bindegewebige Theil eine weiche Consistenz aufwies?

Ein Zufall wies mir den richtigen Weg. Ich behandelte Schnitte von einem excidirten Stücke der Portio, welches nach der Methode von Benda in 10 proc. Salpetersäure und dann in Müller'scher Lösung gehärtet war, zur raschen Färbung mit Anilinessigsäure (einer Lösung von Fuchsin in 2 proc. Essigsäure), spülte die Schnitte kurz in Wasser ab und legte sie in eine 50 proc. Kali aceticum-Lösung. Boi der mikroskopischen Betrachtung der so gewonnenen Präparate war ich überrascht von der Menge der elastischen Fasern, welche in denselben enthalten waren.

Diese Methode liess mich bei Schnitten von anderen Präparaten im Stiche. Ich wandte darauf die von Unna, Herxheimer, Lustgarten, Martinotti und Ferria für die Färbung von elastischen Fasern angegebenen Methoden an. Mit der Methode von Lustgarten erzielte ich stets ein negatives Resultat. Bei der Methode von Herxheimer war mir die Differenzirung der elastischen Fasern von gewöhnlichen Bindegewebsfasern nicht scharf genug. Auch mit der Methode von Unna hatte ich Misserfolge, ebenso gelang mir die Martinotti'sche ${ }^{1}$ )

1) Zeitschrift für wissenschaftliche Mikroskopie 1887. 
Methode nicht bei in Alcohol gehärteten Präparaten, obgleich ich mich ganz genau an die diesbezüglichen detaillirten Angaben von Ferria $\left.{ }^{1}\right)$ hielt. Schliesslich kam ich nach den verschiedensten Versuchen zu einem Verfahren, welches in allen Fällen eine Färbung der elastischen Fasern erzielte, einerlei, ob die betreffenden Stücke vorher in Alcohol, Müller'scher Lösung oder in Wickers heimer'scher Flïssigkeit und dann in Alcohol gelegen hatten. Ich brachte die Schnitte aus Wasser auf 48 Stunden in 2 proc. Kalilauge, spülte sie in Wasser ab und legte sie für 24 Stunden in Anilinessigsäure. Sodann wurden die Schnitte für ein paar Secunden in Wasser hin und her geschwenkt, um darauf in einer 50 proc. Lösung von Kali aceticum conservirt zu werden. Manchmal trat die Färbung der elastischen Fasern bei dieser Methode erst nach 12 bis 24 Stunden klar hervor. Da sich bei diesem Verfahren die Schnitte stark kräuselten und die ganze Structur des Gewebes Schaden litt, so ging ich auch von dieser Methode wieder $a b$, um zu der Methode von Unna und Martinotti zurückzukehren.

Sehr wesentlich erscheint mir bei beiden Methoden die vorausgeschickte Härtung der Objecte in 0,2 proc. Chromsäure. Nach vollendeter Härtung schnitt ich die Objecte mit dem Gefriermikrotom. Die Schnitte kamen sodann auf 24 Stunden in destillirtes Wasser. Ich färbte einen Theil derselben nach $\mathrm{Unn}^{2}{ }^{2}$ ), den anderen nach Martinotti. Bei der Unna'schen Methode schien es mir vortheilhafter, die Färbung mit Vesuvin erst nach der Fuchsinfärbung vorzunehmen. Es kamen also die Schnitte auf 24 Stunden direct in folgende Lösung:

Fuchsin 0,5,

Aq. dest.

Alcohol aa 25,0 ,

Ac. nitr. $(25 \%) 10,0$.

Darauf:

$2-3$ Secunden in 25 proc. Salpetersäure,

10 Minuten in schwaches Essigwasser,

2 Stunden in eine alcobolische Vesuvinlösung (Vesuvin. 2,0, Alcohol. absol. 40,0, Aq. dest. 60,0, coque, filtra, adde Ac. carbol. gutt. III),

1) Zeitschrift für wissenschaftliche Mikroskopie 1888, S. 341.

2) Síehe v. Kahlden, Technik der histologischen Untersuchung pathologisch-anatomischer Präparate. Jena 1890. 
je 5 Minuten in 2 Schälchen mit absolutem Alcohol, 10 Minuten in Cedernöl,

Kanadabalsam.

Für das Gelingen der Methode von Martinotti ist die Qualität des Saffranin sehr wesentlich. Meine ersten Misserfolge mit dieser Methode lagen an der schlechten Beschaffenheit des aus der Charité-Apotheke bezogenen Saffranin. Nachdem ich aus der Fabrik von Dr. Schuchardt in Görlitz das Saffranin 0 erhalten, bekam ich stets positive Resultate. Die Schnitte kamen auf 48 Stunden in eine Lösung von $5 \mathrm{~g}$ Saffranin auf 100 Alc. abs., welcher nach einigen Tagen $200 \mathrm{~g}$ Wasser zugesetzt worden war. Sodann wurden sie gründlich (12 Stunden) ausgewaschen, 24 Stunden lang in absolutem Alcohol ausgewässert, in Bergamottöl aufgehellt und in Damarlack conservirt. Häufig erhielt ich noch schönere Bilder, wenn ich nach dem Auswaschen die Schnitte für einen Augenblick in Glycerin brachte und dann in einer 50 proc. Lösung von Kali aceticum conservirte oder die Schnitte direct aus dem Wasser in der genannten Lösung aufhellte.

Für die mikroskopische Betrachtung erwies sich der Gebrauch des A bbé'schen Condensors ohne Blendung am vortheilhaftesten. Bei beiden eben beschriebenen Methoden erschienen, so betrachtet, die elastischen Fasern blau, das übrige Gewebe braun resp. roth gefärbt.

Durch diese Methoden fand ich die interessante Thatsache, dass die elastischen Fasern der Portio vaginalis in ganz typischer Weise vertheilt sind. Jene beiden gleich grossen Abschnitte der Portio, welche sich schon makroskopisch durch ihre verschiedene Consistenz und mikroskopisch durch den Reichthum glatter Muskelfasern im centralen Abschnitte von einander unterscheiden, grenzen sich nämlich weiter dadurch scharf gegen einander $a b$, dass der periphere Abschnitt sehr reichlich elastische Fasern enthält, während dieselben im centralen Abschnitte fast gänzlich fehlen.

Ich lasse nun zunächst die genaue Beschreibung einer durch Amputation gewonnenen Portio folgen, welche meiner Ansicht nach die normale Vertheilungsweise der elastischen Fasern aufweist. Bei normalen Portionen aus dem geschlechtsreifen Alter fand ich stets dasselbe Bild.

1) Zeitschrift für wissenschaftliche Mikroskopie 1887. 
Das excidirte Stück der vorderen Lippe misst auf einem Sagittalschnitte in der Dicke $1,7 \mathrm{~cm}$, in der Höhe $1,1 \mathrm{~cm}$. Die Länge der excidirten Cervicalschleimhaut beträgt $0,6 \mathrm{~cm}$. Schon makroskopisch erkennt man zwei durch verschiedene Färbung charakterisirte $A b$ schnitte, einen inneren (nach der Cervicalschleimhaut hin gelegenen) braun gefärbten und einen äusseren, der einen blauen Farbenton aufweist. Beide Abschnitte sind gleich gross, sie messen also an der Basis des excidirten Stückes 0,8 bis $0,9 \mathrm{~cm}$. Bei der mikroskopischen Betrachtung erkennt man, dass der blau gefärbte äussere Abschnitt die blau gefärbten elastischen Fasern enthält, während dieselben in dem inneren Abschnitte fast vollständig fehlen. Die Vertheilung der elastischen Fasern ist so, dass sich zunächst unter dem $1 / 2 \mathrm{~mm}$ dicken Plattenepithelsaume eine aus mehrfachen Fasern zusammengesetzte Schicht elastischen Gewebes befindet, welche Ausläufer in die Papillen hineinschickt und welche sich nach der Tiefe hin in ein Netzwerk von Fasern auflöst. An der Basis und nahe der Basis des excidirten Stïckes geht dieses feine Maschenwerk continuirlich in ein sehr reichlich entwickeltes Netzwerk dickerer elastischer Fasern über, welches die Gefüsse umspinnt (Taf. XI, Fig. 1). Mehr nach dem Muttermunde hin steht das oberflächliche mit dem tiefen Netze elastischer Fasern nur durch langgestreckte fadenförmige Ausläufer in Verbindung. Das tiefe Fasernetz endet etwa 2,5 $\mathrm{mm}$ von der Spitze des excidirten Stïckes entfernt. Von der Spitze des excidirten Stückes setzt sich das Plattenepithel noch circa $7 \mathrm{~mm}$ weit fort (Fig. 2). Mit dem Plattenepithel reicht bis zum Beginne der Cervicalschleimhant das oberflächliche Netz elastischer Fasern. In der letzten, nach der Cervicalschleimhaut hin gelegenen Partie von circa 2,5 mm Länge ist dasselbe allerdings nur noch sehr schwach entwickelt, seine Continuitat an einzelnen Stellen unterbrochen (Taf. XII, Fig. 3). Die Cervicalschleimhaut beginnt mit einer 2,6 mm langen Drüse (Nr. 8 auf Fig. 3), an deren nach dem Plattenepithel hin gerichteten Seite langgestreckte, feinste elastische Fasern sich etwa 2,4 $\mathrm{mm}$ weit herab verfolgen lassen. (Fig. 3 ist die directe Fortsetzung von Fig. 2 nach der Cervicalschleimhaut hin.)

Der Fundus der Drïse und die andere Seite wird also nicht mehr von elastischen Fasern eingefasst. Unter dem Plattenepithel liegt eine Reihe von Drüsen, von denen die fünf äusseren von einem gut entwickelten Netzwerk elastischer Fasern umgeben sind, an der sechsten Drüse reichen elastische Fasern auf beiden Seiten etwa bis zur halben Länge der Drüse herunter und an der siebenten Drüse, die nicht bis an den Plattenepithelsaum heranreicht, fehlen sie ganz.

In dem inneren Abschnitte der Portio sieht man hier und da feine, die Muskelfasern begleitende elastische Fasern, die theilweise auch von innen her bis an den Fundus der Cervicaldrüsen heranstreichen. In der Cervicalschleimhaut selbst sieht man nur hier und da einzelne, ganz kurze elastische Fasern.

Die Basis der hinteren Lippe stellt auf einem Sagittalschnitt einen nach oben hin convexen Bogen dar, dessen Sekante $1,7 \mathrm{~cm}$ misst, die Höhe beträgt $1,9 \mathrm{~cm}$. Auch hier grenzen sich makrosko- 
pisch durch die Färbung zwei Schichten von einander ab, von denen die äussere, blau gefärbte Schicht in der Sekante $0,8 \mathrm{~cm}$ misst. Diese äussere Schicht enthält in derselben schon bei der vorderen Lippe beschriebenen Anordnung ein tiefes und ein oberflächliches Netz elastischer Fasern. Auch hier setzt sich ein dicker Plattenepithelsaum uiber eine Reihe von Drüsen hinweg fort. Diesem Saume folgt die oberfächliche Schicht elastischer Fasern, ohne jedoch eine von den Drüsen vollständig zu umspinnen; man sieht nur bier und da feine Auslänfer elastischer Fasern sich eine Strecke weit an den Drüsen herab erstrecken. In dem inneren Abschnitte des Schnittes finden wir ebenfalls feine, mit den Muskelfasern gleichlaufende elastische Fasern. In der Cervicalschleimhaut selber sieht man nur hier und da in der nächsten Umgebung der Gefässe elastische Fasern, die aber nicht zusammenhängen, also nicht wie in der äusseren Schicht eiu continuirliches Netzwerk bilden. Von den die Muskelnfasern umspinnenden elastischen Fasern sieht man bei schwächerer Vergrössernng (Zeiss A. A., Ocular 2) gar nichts. Von den in der Cervicalschleimhaut gelegenen elastischen Fasern sieht man bei derselben Vergrösserung nur diejenigen, welche die grösseren Gefässe umgeben.

In dem beschriebenen Präparate haben wir also eine Portio vor uns, welche schon makroskopisch in zwei ungefähr gleich grosse Abschnitte zerfällt, einen dem Cervicalkanal angrenzenden, von derber Consistenz, mit reichlicher glatter Muskulatur, aber fast ohne Spur von elastischen Fasern, und einen grösseren Abschnitt, der seine weiche Consistenz, sein starkes Retractionsvermögen bei Amputation der Portio seinem Reichthume an elastischen Fasern verdankt. Bei Hunderten von Uteri, bei welchen ich auf diese Verhältnisse speciell geachtet habe, habe ich gefunden, dass in der überwiegenden Mehrzahl der Fälle die beschriebenen zwei Abschnitte ungefähr gleich gross sind. Ich darf daher wohl dieses Verhalten als das normale ansehen und glaube, dass sich hierdurch wichtige Fragen der Physiologie, Pathologie und Therapie der Geburt erklären lassen. Dieses Verhalten ist ferner, wie schon angedeutet, für die Technik der Portioamputation, vielleicht auch für die Verbreitungsweise des Cancroids der Portio von Bedeutung. Endlich ist gerade das eben beschriebene Präparat im Stande, einen Beitrag zur Entstehung der Erosion zu liefern.

Bezüglich der Physiologie der Geburt scheint mir die geschilderte histologische Zusammensetzung der Portio vaginalis eine exacte Erklärung für die Eröffnung des äusseren Muttermundes, für das Verschwinden des Muttermundssaumes zu gewähren. Dieser Saum wird ja bei Erstgebärenden von der Portio vaginalis allein 
gebildet. Er stellt ein Septum dar, welches die obere schon formirte Hälfte des „Durchtrittsschlauches“ Schröder's von der unteren Hälfte bis auf die kleine Muttermundsöffnung vollständig trennt. Was wird nun aus diesem Septum? - Das ist eine Frage, die noch keine exacte Beantwortung erfahren hat. Ist das Septum in seiner Totalität ein so elastisches Gebilde, dass es durch den Druck der Fruchtblase in seinem Volumen auf ein Minimum reducirt und einfach an die Wand gedrückt wird? Ist also die wenig vorspringende Falte des Braune'schen Durchschnittes die ganze stark comprimirte Portio vaginalis uteri? Sie ist es nicht, da, eine solche Elasticität der Portio vorausgesetzt, die Portio sich nach Aufhören des Druckes, also nach der Geburt, sofort wieder zu ihrer früheren Grösse ausdehnen müsste. Dies ist aber, wie die Betrachtung puerperaler Uteri lehrt, nicht der Fall. Die Portio raginalis ist bei ihnen nur durch einen wenig vorspringenden kleinen Wulst gekennzeichnet.

Dieser Wulst stellt nur einen Theil der Portio dar, die meiner Ansicht nach bei der Geburt theils in den Uterus und theils in die Vagina aufgeht. Die Portio wird in ihrer Substanz selbst getrennt, theils durch den Zug der Uterusmuskulatur von oben her, theils durch den in horizontaler Richtung wirkenden Druck der Fruchtblase oder des vorliegenden Theiles. Hierbei verbleibt der centrale Abschnitt der Portio dem Uterus, der periphere dagegen verschmilzt völlig mit der Vaginalwand (Fig. 4).

Eine solche Entfaltung oder besser Auseinanderfaltung der Portio theils durch Zug, theils durch Druck ist nur möglich und nichts Anderes ist möglich - bei einer derartigen Zusammensetzung der Portio, wie ich sie eben beschrieben habe. Bestände die Portio nur aus elastischem Gewebe, so würde sie, wie schon erwähnt, durch die Fruchtblase einfach zusammengedrückt werden; bestände sie nur aus Muskulatur und Bindegewebe ohne elastische Fasern, so würde der Muttermund sich nur sehr schwer und nur nach vorausgegangenen stärkeren Zerreissungen erweitern, da dieses gewöhnliche Bindegewebe den ursprünglichen Zusammenhang der Muskulatur der Portio mit der Scheide aufrecht zu erhalten suchen würde.

Natürlich werden bei der Auseinanderfaltung der Portio die elastischen Fasern um so stärker angespannt, je weiter sie nach der Peripherie hin liegen. Den stärksten Zug werden diejenigen elastischen Fasern erfahren, welche die Verbindung zwischen 
Scheide und supravaginaler Partie des Uterus vermitteln. Ihre Dehnung wird indessen bei normaler Geburt keine übermässige sein, da die Zugwirkung sich auch auf die zahlreichen elastischen Fasern der Scheide selbst überträgt.

Ein Präparat, welches ich von einem kurz nach der Geburt verstorbenen Falle von Placenta praevia aus dem Ende des achten

Fig. 4.
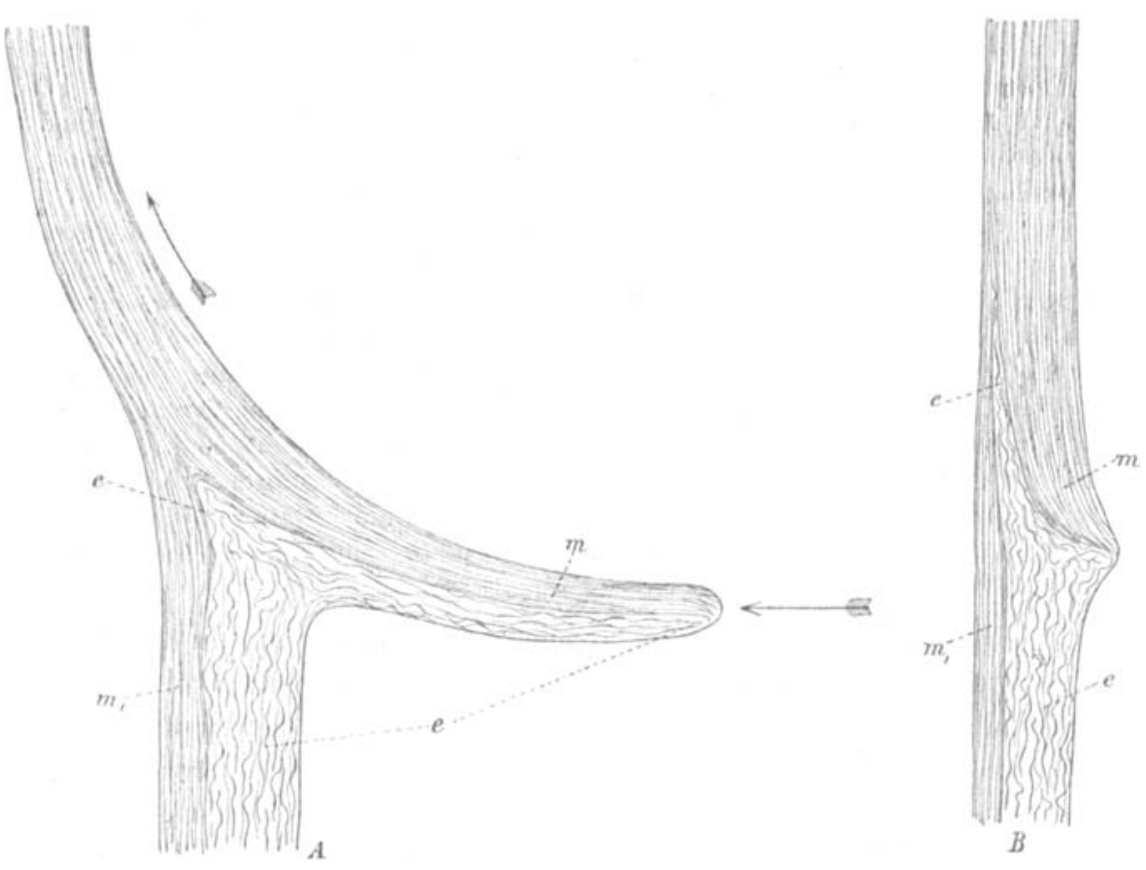

Schematische Darstellung der Verlagerung des peripheren und centralen Abschnittes der Portio bei der Erweiterung des äusseren Muttermundes. Die Pfeile deuten den Zug von oben her und den horizontal wirkenden Druck der Fruchtblase an.

$m=$ glatte Muskulatur des inneren Abschnittes der Portio. $m_{1}=$ glatte Muskulatur der Vaginalwand. $e=$ elastische Fasern des äusseren Abschnittes der Portio und der Vaginalwand.

Monates der Schwangerschaft gewonnen habe, zeigt diese Dehnung und die Rolle, welche die elastischen Fasern dabei spielen, in exquisiter Weise. Ich lasse zunächst die Beschreibung des Präparates folgen (cf. Taf. XII, Fig. 5 u. 6).

Die Dicke der vorderen Lippe beträgt in der Höhe des Scheidenansatzes $7 \mathrm{~mm}$. Nach abwärts überragt sie den Scheidenansatz um $2^{1} / 2 \mathrm{~mm}$. Die Vaginalwand ist in der Höhe des Scheidenansatzes $4 \mathrm{~mm}$ dick. 
An der Lippe unterscheidet man sehon makroskopisch zwei Schichten. Die äussere ist in der Höhe des Scheidengewölbes $2,7 \mathrm{~mm}$ dick und erstreckt sich in gleicher Dicke bis zum unteren Ende der Lippe. Die Scheidenwand und der genannte äussere Abschnitt der Lippe weisen eine durchaus gleiche Zusammensetzung auf. Die Bindegewebsfasern sind zu weitmaschigen Netzen auseinandergezogen, zwischen denen sich elastische Fasern ausspannen. In der Lippe selber sind die elastischen Fasern sehr dünn, nach der Scheide hin werden sie allmälig dicker und bilden hier theilweise Bündel von elastischen Fasern, welche mit den Fasern des gewöhnlichen Bindegewebes zusammenlaufen und durch feinere elastische Fasern mit einander verbunden sind, An der Lippe ist der Uebergang des oberflächlichen, direkt unter dem Epithel gelegenen Fasernetzes in das tiefere Netz ein ganz allmäliger. Die elastischen Fasern der Lippe reichen vom Scheidenansatze bis zum unteren Ende der Portio herunter und lassen sich bis dahin verfolgen, wo das Plattenepithel aufhört (bis zum Beginn der Cervicalschleimhaut). Der innere, dem Cervicalcanal zugewandte Abschnitt der Lippe ist in der Höhe des Scheidenansatzes $4,3 \mathrm{~mm}$ dick. Er enthält keine elastischen Fasern, er besteht zum grössten Theile aus gewöhnlichem Bindegewebe, welches ebenfalls netzförmig angeordnet ist. Allerdings sind die Netze nicht so weit, wie im äusseren Abschnitte. Daneben finden sich in dem inneren Abschnitte dünne Bündel längs verlaufender glatter Muskulatur. Dickere, in Falten gelegte longitudinale Bündel glatter Muskulatur finden sich ausserdem als äussere Begrenzung der Vaginalwand und gehen von dieser auf den Uterus iiber.

Gegenüber dem oben beschriebenen Präparate von einem nicht puerperalen Uterus fallen an dem letzten Präparate zwei Unterschiede in die Augen: die Bindegewebsbündel der peripheren Schicht der Portio sind in ein weitmaschiges Netzwerk ausgezogen, dessen Lücken von elastischen Fasern ausgefüllt werden. Diese Fasern sind beträchtlich, dünner als die von der Portio des nicht puerperalen Uterus. Es sind diese zwei Unterschiede im histologischen Verhalten der Portio bei puerperalem und nichtpuerperalem Uterus nur durch eine bei der Erweiterung dès äusseren Muttermundes erfolgende Dehnung des peripheren elastischen $\mathrm{Ab}$ schnittes der Portio zu erklären.

Berücksichtigt man ferner den Umstand, dass bei dem puerperalen Uterus die periphere Schicht der Portio nur den dritten Theil, nicht, wie bei dem anderen Uterus, die Hälfte der Dicke der Lippe ausmacht, so spricht auch dies dafür, dass ein Theil der peripheren Schicht der Portio mit der Vaginalwand verschmolzen ist.

Auch die Bindegewebsbündel der inneren Schicht der Portio sind in ein, wenn auch engmaschigeres Netzwerk verwandelt. 
Diese letztere Umwandlung kann nicht durch den Druck der Fruchtblase, sondern nur durch den Zug von oben her zu Stande gekommen sein. Denn der Druck der Fruchtblase kann im inneren Abschnitte nur zu einer Verdichtung des Bindegewebes führen.

Auffallend war mir ferner an diesem. Präparate, dass in der inneren Schicht der Portio bedeutend weniger Muskelbündel vorhanden waren, als an dem nichtpuerperalen Uterus. Mit Rücksicht auf die letzte Arbeit Bayer's' ${ }^{1}$ ) bin ich geneigt, diese Differenz durch eine Verschiebung der Muskelfasern nach oben, die nach Bandl und Bayer ${ }^{2}$ ) bereits in der Schwangerschaft erfolgt, zu erklären. Dass die Portio eines kreissenden und puerperalen Uterus arm an glatter Muskulatur ist, betont auch Hofmeier ${ }^{3}$ ) nachdrücklich. Am nichtpuerperalen Uterus dagegen findet sich unter der Cervicalschleimhaut eine gut entwickelte Schicht grösstentheils longitudinal verlaufender glatter Muskulatur.

B and $1^{4}$ ) sagt über die Eröffnung des äusseren Muttermundes Folgendes: „Durch die Verkürzung der Muskulatur, die sich an das Bindegewebe der Vaginalportion inserirt, öffnet und erweitert sich das Ostium externum. Ist das Ostium externum ad maximum erweitert, so versehwindet durch weitere Verkürzung der Muskulatur der noch fühlbare Theil der Vaginalportion in der Wand des Uterovaginalschlauches, so dass schliesslich nur ein feiner Saum derselben fühlbar ist.“ Diese Erklärung deckt sich mit der meinigen. Einen Anspruch auf Richtigkeit kann sie allerdings nur erheben, wenn man zu gleicher Zeit, wie ich es gethan, den Nachweis führt, dass dieses Bindegewebe ausserordentlich reich an elastischen Fasern ist.

Die Frage, die ich oben nur kurz berührt, bedarf einer ausführlicheren Besprechung, nämlich die, ob der äussere Muttermund durch den Zug von oben her, wie Bandl es annimmt, oder durch den Druck der Fruchtblase, wie Hofmeier und Schröder es annehmen, erweitert wird. Hofmeier ${ }^{5}$ ) drückt sich in Bezug auf die Erweiterung des ganzen Cervix folgendermaassen aus: „Entsprechend dieser Anschauung von der mangelhaften Activität des unteren Lterinsegmentes während der Geburt, glaube ich ferner auf Grund meiner klinischen Erfahrungen annehmen zu müssen,

1) Verhandlungen der deutschen Gesellschaft für Gynäkologie. III.

2) Morphologie der Gebärmutter in Freund's „Gynäkologische Klinik“.

3) In Schröder, Der schwangere und kreissende Uterus. Bonn 1886.

4) Dieses Archiv, Bd. XII, S. 340.

5) 1. c. 
dass die Erweiterung des inneren Muttermmdes bei der Geburt weniger durch ein actives Auseinanderziehen oder Gezogenwerden, als durch ein passives Auseinandergetriebenwerden von Seiten der Blase oder des vorliegenden Theiles erfolgt. Es ist freilich das Eine ohne das Andere kaum zu denken, da natürlich bei der Contraction das untere Segment nothwendig gespannt und somit ein gewisser Zug auf die Theile um den inneren Muttermund ausgeübt wird; fehlt aber ein vorliegender Theil, der zu gleicher Zeit von inwendig keilartig gegen den inneren Muttermund getrieben wird, so bleibt die Eröffnung des inneren Muttermundes stets eine mangelhafte und unvollkommene, wie dies die Fälle von frühzeitigem Blasensprunge bei engem Becken und Blasenmole z. B. lehren. Es wird deswegen im Ganzen auch nach meiner Erfahrung erst bei stärkeren mechanischen Hindernissen, wenn also der Zug des contractilen Theiles des Uterus auf das untere Segment und durch dieses auf den Cervix ein erheblicher und lang andauernder gewesen ist, der Cervix über den Beckeneingang hinaufgezogen, während er für gewöhnlich seine Stellung im kleinen Becken beibehält. Bei Erstgebärenden ist wohl meist der Widerstand des Cervix und äusseren Muttermundes an und für sich so gross, dass derselbe über die Beckeneingangsebene hinaufgezogen wird, wenigstens vorn. Bei Mehrgebärenden aber und normalem Becken behält der Cervix etwa seine Stelle im kleinen Becken bei und wird grösstentheils auseinandergedrückt." Im Schröder'schen Lehrbuche (11. Auflage, S. 173) finden wir in Bezug auf diese Frage folgenden Passus: „Für die Erweiterung des inneren, besonders aber für die des äusseren Muttermundes ist die die Theile auseinanderdrängende Fruchtblase von grosser Bedeutung, da ein eigentlicher Zug auf den äusseren Muttermund gar nicht mehr ausgeübt wird. Springt die Blase vorzeitig, so geht auch die Erweiterung des Cervix langsamer von statten; ja, in Fällen, in denen die bereits keilförmig in den Cervix dringende Blase springt, fällt derselbe wieder deutlich zusammen, zum Zeichen, dass seine Erweiterung ganz wesentlich durch das Hineindrängen der Blase bedingt war - Collaps des Muttermundes."

Meine Ansicht über diese Frage geht dahin, dass der äussere Muttermund nur durch das harmonische Ineinandergreifen beider Kräfte, des Zuges von oben her und des Druckes seitens der Fruchtblase oder des vorliegenden Theiles, so erweitert wird, dass sein Saum völlig in den Genitalschlauch aufgeht. So wie Hofmeier und Schröder darauf hinweisen, dass bei Fehlen der 
Blase oder eines vorliegenden Theiles der Muttermund sich nicht völlig erweitert, so kann ich den Beweis erbringen, dass auch die Wirkung der Fruchtblase allein ein völliges Aufgehen der Portio vaginalis in den Genitalschlauch nicht zu. Wege bringt.

Bei mangelhaft erweitertem Cervix Mehrgebärender, wo eine rasche Entbindung indicirt war, habe ich mehrmals nach dem Vorschlage Mäurer's ${ }^{1}$ ) einen dünnwandigen Kolpeurynter in den Uterus eingelegt, ihn bis zur Grösse eines Kindskopfes mit Wasser angefüllt und ihn dann durch Zug am Schlauche aus dem Uterus herausgezogen. Es ahmte somit dieses Verfahren die Wirkung der Blase resp. des vorliegenden Theiles nach. Der einzige, für diese Frage aber ganz unwesentliche Unterschied lag in dem verschiedenen Angriffspunkte der Kraft. Niemals kam es in den Fällen, wo ich die Methode angewandt, zu einem Aufgehen der Portio in die Wand des Genitalschlauches. Die Portio blieb vielmehr als solche bestehen, der Muttermundssaum wies eine Breite von ca. zwei Fingern auf, nur wurde dieser Saum fest gegen die Vaginalwand angedrückt. Es war somit durch dieses Verfahren der ganze Cervix in ein cylindrisches Rohr von solcher Weite verwandelt, dass die kindskopfgrosse Blase hindurch konnte. Dieses cylindrische Rohr steckte mit seinem unteren Ende, der Portio vaginalis, in dem Scheidenrohre - also es kam nicht zu dem einen „Durchtrittsschlauche" Schröder's, sondern zu einer Einschachtelung zweier Röhrensysteme (s. Fig. 7, S. 272). ${ }^{2}$ )

Auf Grund dieser Befunde glaube ich also, dass die Blase oder der vorliegende Theil allein, wohl gelegentlich, nämlich bei grosser Elasticität der Portio, den äusseren Muttermund so weit dilatiren können, dass der Kindskörper hindurchgeht. Allein hier-

1) Centralblatt für Gynäkologie 1887, Nr. 25.

2) Bezüglich des therapeutischen Werthes des Verfahrens von Mäurer lässt sich also aus meinen Beobachtungen der Schluss ziehen, dass es den yon dem Autor für alle Fälle als sicher angenommenen Erfolg nur in den Fällen babes kann, wo die Elasticitat der Portio gross genug ist, un zu einex ringförmigen Oeffinung von $11 \mathrm{~cm}$ Durchmesser ausgeweitet zu werden. Auf eine Erweiterung des Muttermundes, wie sie bei der normalen Geburt stattfindet, ist dagegen nicht mit Sicherheit zu rechnen, weil durchaus nicht immer so rasch, wie Mäurer es in seinem Falle beobachtete, Wehen eintreten. In Fällen von Rigidität der Portio wird somit das Verfahren nicht zum Ziele führen oder sehr bedeutende Zerreissungen setzen. Kleinere Zerreissungen bleiben selbst bei dehnbarem Muttermundssaume nicht aus. Immerhin ist das Verfabren für eine grosse Zahl von Fällen als eine werthvolle Bereicherung der geburtshülflichen Therapie zu betrachten. 
bei wird der von der Portio vaginalis repräsentirte Muttermundssaum einfach gegen die Vaginal- und gegen die Beckenwand vorgedrängt, ein Aufgehen der Portio vaginalis in den Genitalschlauch, wie es doch das Normale ist, findet nicht statt. Hierzu ist eben noch eine zweite Kraft, die Zugkraft von oben her, erforderlich.

Fig. 7.

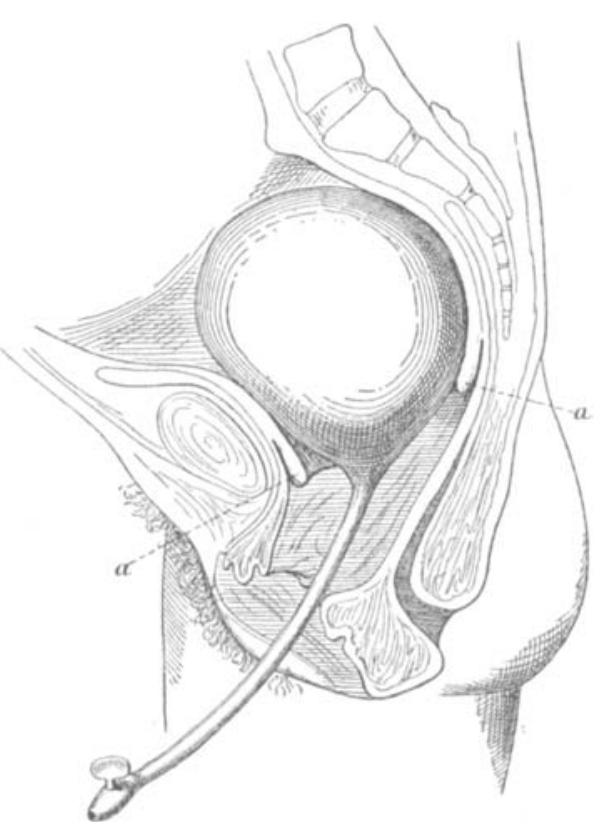

Noch ein Punkt bedarf der Erledigung. Nehmen wir den Muttermundssaum bei einer I para $\mathrm{zu} 6 \mathrm{~cm}$ Länge an, so würde durch die Auseinanderfaltung dieses Saumes, welcher der Portio vaginalis entspricht, ein Canal von $12 \mathrm{~cm}$ Länge entstehen. Dies ist nun in Wirklichkeit nicht der Fall und rührt daher, dass Hand in Hand mit der Auseinanderfaltung der Portio auch eine Retraction der beiden Abschnitte der Portio erfolgt. Man kann diese Retraction - vielleicht ist es auch eine active Contraction der Muskulatur des Cervix - beobachten, wenn man bei einer I para den Muttermund mit den Fingern auseinanderdrängt. Es legt sich dann der Muttermundssaum nicht etwa in Falten, sondern er verdickt sich. Ebenso werden nach Incisionen dieses Saumes die resultirenden Lappen bedeutend kürzer, dafür aber dicker. 
Nach der Geburt findet gewöhnlich keine völlige Wiederherstellung der Form der Portio statt. Indessen sind die individuellen Unterschiede hier sehr gross. Es giebt Personen, bei denen aus der Beschaffenheit der Portio und des Muttermundes allein ein sicheres Urtheil über eine stattgehabte Geburt nicht zu fällen ist. Bekannt ist ja diese Thatsache für die Fälle, wo schon viele Jahre seit der letzten Geburt verstrichen sind. Indessen findet man eine Portio fast wie bei einer I para auch bei manchen Individuen, die erst vor kurzer Zeit geboren haben. Für diese dürfte wohl die Annahme gerechtfertigt sein, dass ihre Portio reich an elastischen Fasern ist. Bei denselben Individuen findet man gewöhnlich auch eine fast virginelle Scheide, einen intacten Damm - also ebenfalls Zeichen einer guten Elasticität dieser Gebilde. Gerade das Gegenstück zu diesen Fällen bilden Frauen, die oft rasch hintereinander und ohne genügende Erholung in der Zwischenzeit geboren haben. Bei diesen kann die Portio völlig verschwinden, so dass sich die Scheide direct am äusseren Muttermund inserirt, also Verhältnisse persistiren, wie sie für die zweite Periode der Geburt charakteristisch sind. Ist in solchen Fällen eine Erosion vorhanden, so liegt diese im Scheidengewölbe. Hier liegt der Gedanke nahe, dass die späteren Geburten bei noch gedehnten elastischen Fasern stattfanden und dass hieraus eine Ueberdehnung resultirte.

Als Beispiel für das Gesagte führe ich folgenden Fall an:

Frau H., 32 Jahre alt, hat in ibrer siebenjährigen Ehe fünf ausgetragene Kinder geboren und eine Fehlgeburt durchgemacht. Sie klagt über Kreuzschmerzen, Schmerzen über den ganzen Leib und Vorfallsbeschwerden.

Bauchdecken, Ligamente und Beckenboden sehr schlaff. Vulva klaffi, Senkung der vorderen Vaginalwand, Uterus anteflectirt, vergrössert, sehr beweglich. Die vordere Lippe ragt wenig, die hintere gar nicht in die Seheide hinein, so dass ein hinteres Scheidengewölbe gar nicht existirt und die hintere Scheidenwand sich vom äusseren Muttermunde aus in einer horizontalen Ebene nach hinten erstreckt, um dann nach unten umzubiegen. Der horizontale Abschnitt ist von einer folliculären Erosion eingenommen, die nur durch eine höchstens $2 \mathrm{~mm}$ dicke Schicht von dem sie überdachenden Douglas'schen Raume getrennt wird. Zieht man jedoch die Portio mit einer Kugelzange an, so hat man das charakteristische Bild einer stark hypertrophischen Portio mit leicht blutender Erosion.

Therapie: Curettement, Excision der Erosion, Law on Tait.

Mit Rücksicht darauf, dass im Allgemeinen bei Mehrgebärenden die Auseinanderfaltung der Portio, wie sie in der EröffnungsArchir f. Gynaksologie. Bd. XLI. Hft, 1. u. 2. 
zeit stattfindet, in gewissem Grade fortdauert, wird natürlich bei späteren Geburten die Eröffnung des äusseren Muttermundes nur geringe Schwierigkeiten machen.

Uebrigens finden wir die beschriebene Auseinanderfaltung der Portio, wie sie normaliter nur bei der Geburt vorkommt, auch bei gewissen pathologischen Verhältnissen ausgeprägt. Sowohl wenn der Uterus abnorm stark nach oben, als auch wenn die Scheide abnorm stark nach unten gezogen wird, tritt jene Auseinanderfaltung der Portio ein, so dass sich die Scheide direct am Muttermunde inserirt. So finden wir manchmal bei intraligamentären Tumoren, die den Uterus eleviren, oder bei Retroflexio uteri gravidi im Scheidengewölbe keine Andeutung der Portio, sondern nur die Oeffnung des Muttermundes. So sehen wir, dass bei Prolaps der Vagina mit Elongatio colli die stark verdickte Vaginalwand im Niveau des äusseren Muttermundes auf den Uterus übergeht. Die hierbei stets zu beobachtende Verdickung der Vaginalwand braucht durchaus keine Folge der Hypertrophia colli zu sein, sondern sie lässt sich auch dadurch erkiären, dass dieser verdickte Abschnitt der Vaginalwand früher einen Abschnitt der Portio darstellte.

Wenn ich für eine normale Erweiterung des äusseren Muttermundes das elastische Fasernnetz der peripheren Schicht der Portio für sehr wichtig angesprochen habe, so liegt damit auch der Schluss sehr nahe, dass gewisse Störungen in der Erweiterung des Muttermundes durch einen Mangel der elastischen Fasern in der Portio bedingt sind - Störungen, welche man bis jetzt auf eine anatomisch nicht näher charakterisirte Rigidität des Muttermundssaumes bezog. Eine solche Rigidität findet man in der Regel bei alten Erstgebärenden. Und zwar handelt es sich hierbei meiner Ansicht nach entweder um eine mangelhafte Entwicklung der elastischen Fasern, um ein Stehenbleiben auf einer kindlichen Stufe der Entwicklung oder um einen Altersschwund der elastischen Fasern.

In die erste Kategorie gehören die Fälle, wo trotz der Ausübung des Coitus mit einem gesunden Manne die Conception erst nach langjährigem geschlechtlichen Verkehre erfolgte. Die Ursache der Sterilität liegt hier in einer mangelhaften Entwicklung des Gesammtorganismus, wie auch des Geschlechtsapparates, welche die Frauen zunächst für das Fortpflanzungsgeschäft untauglich macht. Es handelt sich hier um die Frauen mit infantilem Ha- 
bitus, um das , ,mangelhaft entwickelte Weib“; dessen Typus von W. A. Freund ${ }^{1}$ ) bis in das kleinste Detail beschrieben worden ist.

Die Rigidität oder Starrheit der Portio bei diesen Individuen betrachtet auch H. W. Freund ${ }^{2}$ ) als angeboren, ohne sich indessen über das die Rigidität bedingende Moment zu äussern. W. A. Freund ${ }^{3}$ ) sieht dagegen die Ursache der Rigidität in einer Entzündung der Portio. Er sagt hierüber Folgendes:

„Hervorheben möchte ich der praktischen Bedeutung wegen die Kürze and schwierige Entfaltbarkeit des Cervix bei im Ganzen in der Ausbildung zurückgebliebenem, congenital anteflectirtem Uterus von Personen, welche an chronischem Vaginal- und Cervicalkatarrh leiden, und älteren Erstgebärenden mit folliculärer Durchsetzung des Cervix."

Auch in dem Schröder'schen Lehrbuche (11. Aufl., S. 551) finden wir dieselbe Ansicht: „Die Rigidität des Muttermundes ist bedingt durch chronisch entzündliche Processe des Uterus und vorzugsweise des Cervix."

P. Müller beschuldigt in seinem Handbuche der Geburtshülfe eine Hypertrophie der Portio für das Zustandekommen der Rigidität.

Für die Fälle von Prolaps mögen beide Deutungen zutreffend sein, für die Fälle von Rigidität bei alten Erstgebärenden sind sie das nicht. Denn bei diesen handelt es sich nicht, wie die genannten Autoren annehmen, um eine Rigidität des ganzen Cervix, sondern nur um eine Rigidität der Portio vaginalis. Die supravaginale Partie des Cervix wird schon, ganz im Gegensatze zu dem Geburtsverlaufe bei Prolaps, frühzeitig entfaltet, und nur die Portio bleibt als derber, dicker Saum bestehen. Dass die Entzündung oder Hypertrophie aber nur die Portio betreffen sollte, dass sie genau an der Verbindung mit der Scheide aufhören sollte, kann man doch nicht annehmen. Zudem kam $\mathrm{Walich}^{4}$ ) in zwei Fällen von Rigidität der Portio, die eine ernste Geburtsstörung setzte, bei der mikroskopischen Untersuchung aus der Portio excidirter Stücke zu dem Resultat, dass das Collum keine essentiellen Veränderungen zeigte, welche auf die anatomische Rigidität zu beziehen seien.

1) Gynäkologische Klinik.

2) Zeitschrift für Geburtshülfe, Bd. XVI, S. 11.

3) a. a. $0 .$, S. 138.

4) Archives de tocologie 1890, p. 182. 
In dem einen Falle langer Stillstand der Geburt, Incisionen des Collum, Zerreissung desselben, Basiotripsie (Porak) durch die Ruptur hindurch. Im zweiten Falle nach Incisionen spontane Geburt.

Walich sieht daher die Ursache der mangelnden Erweiterung nur in Wehenschwäche begründet. Dem gegenüber bemerkt mit Recht Porak ${ }^{1}$ ), dass die klinischen Thatsachen auf gewisse primitive Läsionen des Collum in Fällen von Rigidität hinwiesen. Denn die Untersuchung ergiebt in gewissen Fällen mit Bestimmtheit eine Veränderung in der Consistenz und der Ausdehnungsfähigkeit des Cervicalgewebes. „Zweifelsohne ist", wie Porak fortfährt, ,die Natur dieser Veränderungen noch absolut unbekannt, allein das Studium der anatomischen Vorgänge bei der Erweichung des Collum - eine noch sehr dunkle Frage - wird die Ursache der Rigidität auffinden lassen."

Walich hat insofern Recht, als die elastischen Fasern bei der gewöhnlichen Herrichtung mikroskopischer Schnitte aus der Portio dem Auge völlig entgehen. Dass die elastischen Fasern aber gewisse Phasen der Entwicklung und Rückbildung durchmachen, dass Störungen dieșer Vorgänge stattfinden können, beweisen folgende Präparate:

1) Portio eines neugeborenen Kindes:

Die vordere Lippe ist $0,9 \mathrm{~cm}$ lang und $0,3 \mathrm{~cm}$ dick. Von den inneren Schichten der Vagina aus setzt sich dicht unter dem Plattenepithel eine Schicht elastischer Fasern auf die Portio fort, um genau da zu enden, wo das Cylinderepithel anfängt. Bei schwächerer Vergrösserung (Zeiss AA, Ocular 2) sieht man, dass diese Schicht eine Dicke von $1 / 2 \mathrm{~mm}$ hat. Sie besteht aus langgestreckten, parallelen, elastischen Fasern, welche erst gegen die Spitze der Portio hin sich in ein Netzwerk von Fasern auflösen. Auch bei stärkerer Vergrösserung (Zeiss DD, Ocular 2) sieht man, dass eine zusammenhängende Schicht elastischer Fasern in der genannten Entfernung vom Plattenepithel aufhört und dass an diese sich eine an Muskelfasern reiche Schicht nach innen hin anschliesst. Parallel zu den Muskelfasern laufen vereinzelte elastische Fasern. Nur um die grösseren Gefässe dieser an Muskelfasern reichen Schicht sieht man noch eine mehrfache Schicht elastischer Fasern, die aber nicht untereinander zusammenhängen (Taf. XII, Fig. 8).

Aus dieser Beschreibung geht also hervor, dass dem Neugeborenen das tiefe Netzwerk elastischer Fasern, wie es für die völlig ausgebildete Portio charakteristisch ist, vollständig fehlt. Bleibt dieser Zustand bestehen, so ist dadurch gewiss eine mangel-

1) Annales de gynécologie, Bd. XXXIV, S. 390. 
hafte Eröffnung des Muttermundes erklärt. Dass in der Ausbildung des elastischen Fasernetzes grosse Differenzen vorkommen, kann ich durch ein instructives Präparat beweisen.

2) Vordere Lippe einer 25jährigen, seit acht Jahren steril verheiratheten Frau, die an Pneumonie gestorben war.

Die Portio fühlte sich sehr derbe an, der Uterus war nur $5 \frac{1}{2} \mathrm{~cm}$ lang. Die Adnexe waren völlig normal bis auf das linke Ovarium, welches in eine wallnussgrosse, mit einem Blutgerinnsel gefüllte Cyste verwandelt war. Der Befund an der vorderen Lippe war folgender: Die Dicke derselben in der Höhe des Scheidenansatzes beträgt $11 \mathrm{~mm}$. Nach abwärts überragt sie den Scheidenansatz um $7 \mathrm{~mm}$. Die Vaginalwand ist $2 \mathrm{~mm}$ dick und setzt sich mit einem $3 \mathrm{~mm}$ breiten Streifen, welcher bis zur Spitze der Lippe herabreicht, in den Uterus hinein fort. Dieser Streifen, welcher schon makroskopisch durch seine hellere Färbung kenntlich ist, charakterisirt sich mikroskopisch durch seinen Gehalt an elastischen Fasern. Dieselben bilden ein oberflächliches, unter dem Plattenepithel gelegenes Netzwerk und ein tiefes, die Gefässe umspinnendes Netz mit äusserst zarten Fasern, welche nur gegen die Uebergangsstelle auf die Vagina dicker werden. Der dem Cervicalcanale angrenzende $8 \mathrm{~mm}$ breite Abschnitt enthält Bindegewebe und glatte Muskulatur, aber keine elastischen Fasern.

An diesem Präparate, das von einem infantilen Uterus stammt, ist also Zweierlei hervorzuheben. Einmal ist die periphere, die elastischen Fasern enthaltende Schicht sehr dünn: Sie nimmt nur wenig über den vierten Theil der Gesammtdicke der Lippe ein, während normaliter die halbe Dicke der Lippe auf diese Schicht entfallen soll. Ferner sind die elastischen Fasern durchweg viel dünner und zarter als an dem ersten oben beschriebenen Präparate.

3) Portio einer 72jährigen Person mit Schwund der elastischen Fasern, durch Amputation (wegen Prolaps?) gewonnen.

Fig 9.

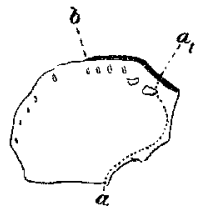

Fig. 10.

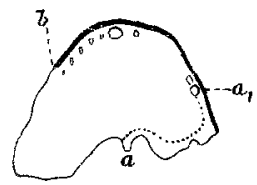

Ich verdanke dieselbe der Güte des Herrn Privatdocenten Dr. Döderlein.

Die beistehenden Zeichnungen sind die Sagittalschnitte durch beide Lippen in natürlicher Grösse. Der Bezirk der elastischen Fasern liegt zwischen $a$ und $a_{\text {, }}$, und zwar findet sich hier nur ein ganz schmaler Saum theils sehr dünner und keineswegs continuirlich zusammenhängender elastischer Fasern theils von dicken kurzen Fasern, 
welche von einem gemeinschaftlichen Centrum aus nach allen Seiten wie die Fangarme eines Polypen ausstrahlen und frei enden. Die punktirte Linie von a bis $a$, deutet diesen Saum an. Nach innen zu enden die Fasern also ungefähr in der Mitte zwischen Portio- und Cervicalschleimhaut, nach unten hören die elastischen Fasern an der ersten abgeschnürten und von Plattenepithel überzogenen Cervicaldrüse bei $a$ auf. Ich werde weiter unten noch darauf einzugehen haben, weswegen ich die sämmtlichen von $a$, bis $b$ unter dem Plattenepithele gelegenen Drüsen nicht als Erosionsdrüsen bezeichne, sondern ihre Abstammung von der Cervicalschleimhaut herleite.

Die Merkmale also, durch welche sich diese senile Portio von einer jugendfrischen unterscheidet, sind sehr auffällige. Das Hauptmerkmal ist der völlige Schwund der elastischen Fasern in dem grössten Theile der Portio. Während wir gezeigt haben, dass bei jungen Personen die elastischen Fasern normaliter die äussere Hälfte der Portio einnehmen, also etwa den Bezirk zwischen $a, a, b$, so sehen wir von diesem grossen Bezirke nur noch einen ganz schmalen Saum elastische Fasern aufweisen - und zwar in nächster Nähe des Ueberganges der Scheide auf die Portio.

Es fehlt ferner das reich entwickelte oberflächliche und tiefe Netzwerk elastischer Fasern. Die Fasern selbst sind durchweg dünner.

Auch die polypenähnlichen Knäuel ${ }^{1}$ ) elastischer Fasern kommen in einer jüngeren Portio nicht vor. Ich sah sie noch in einer anderen Portio einer alten Frau, deren Uterus wegen Carcinom von Herrn Geheimrath Gusserow exstirpirt worden war. Eine weitere Beschreibung der Vertheilung der elastischen Fasern in diesem zweiten Falle zu geben, bin ich nicht im Stande, da dieses Präparat nicht in der richtigen Weise gehärtet war und die Härtung der feineren elastischen Fasern daher mangelhaft ausfiel.

Die erwähnte Knäuelbilḍung liesse sich vielleicht in Beziehung zur senilen Umbildung der Portio bringen. Vielleicht nimmt die Elasticität der elastischen Fasern selbst ab. Es kommt

1) Während der Drucklegung meiner Arbeit ist eine Arbeit von Martin B. Schmidt erschienen (,Ueber die Altersveränderungen der elastischen Fasern in der Haut." Virchow's Archiv, Bd. CXXV, Hft. 2, S. 239), welche meine Deutung der „Knäuel" elastischer Fasern als einer senilen Degeneration bestätigt. Schmidt hat dieselben ,Knänel" in der Lippen- und Wangenhaut von Individuen aus den fünfiziger Jahren gesehen und deutet sie als eine hyaline Aufquellung und Schollenbildung durch ungleichmässige Verdickung oder durch Zusammenrollen der gequollenen Fasern - gewiss eine interessante Analogie zwischen Befunden am Mund und Muttermund bei alten Individuen! 
dann zu Continuitätstrennungen an den Stellen, wo die Netze elastischer Fasern nur mit feinen Ausläufern zusammenhängen, und aus zwei zusammenhängenden Netzen werden dann zwei Knäuel. Dieser Vorgang lässt sich vielleicht am besten mit der Zerreissung eines Spinnengewebes vergleichen.

Uebergänge zu dieser Atrophie der elastischen Fasern habe ich in Präparaten von Frauen in den vierziger Jahren gefunden. Der Schwund betraf vornehmlich das tiefe Netzwerk.

Der Altersschwund der elastischen Fasern erklärt, wie ich schon oben andeutete, auch die Rigidität der Portio bei denjenigen alten Erstgebärenden, die nur steril blieben, weil sie nicht cohabitirten, die also nach vollzogener Cohabitation rasch concipirten. Schon gegen die Zeit der Menopause hin nehmen die elastischen Fasern im peripheren Abschnitte der Portio bedeutend ab. Bei einer 40jährigen Person z. B., die mit 39 geheirathet und sofort concipirt hat, ist schon die regressive Metamorphose der elastischen Fasern der Portio im vollsten Gange. Sie beginnt vielleicht schon mit dem 30. Jahre, oder noch früher, falls bis dahin keine Cohabitation und Geburt erfolgte. Hierdurch fallen mächtige Reize fort, welche sicher einen Einfluss auf die Entwicklung resp. das Bestehenbleiben der elastischen Fasern ausüben. Um so grösser wird natürlich die Rigidität sein, wenn der Altersschwund der elastischen Fasern in einer Portio mit von Hause aus mangelhafter Entwicklung derselben vor sich geht - je älter also eine Frau mit infantilem Habitus wird, ohne zu concipiren.

Beruht, wie ich annnehme, die Rigidität der Gewebe, speciell der Portio vaginalis der alten Erstgebärenden auf einem Mangel an elastischen Fasern, so ist auch der Therapie durch die anatomische Betrachtung der einzig richtige Weg vorgezeichnet. Es können in diesen Fällen, wo die Elasticität der Gewebe fehlt, mechanische Dehnungsversuche nichts nützen. Forcirt man sie, so müssen sie zu tiefgehenden Zerreissungen führen. Hier liegt ein Hinderniss vor, welches in Fällen, wo Gefahr für Mutter oder Kind die Vornahme der Entbindung erheischt, nur das Messer zu beseitigen im Stande ist. Die Berechtigung der von Skutsch ${ }^{1}$ ) empfohlenen, von $\operatorname{mir}^{2}$ ) zuerst ausgeführten tiefen Cervixincisionen ist für diese Fälle von Olshausen ${ }^{3}$ ) theoretisch anerkannt, und

1) Dieses Archiv, Bd. XXXI, Hft. 3.

2) Ebendas., Bd XXXVII, Hft. 1 u. Therapeutische Monatshefte 1890, Mai.

3) Centralblatt für Gynäkologie 1890, S. 583. 
mein hochverehrter Chef und Lehrer Herr Geheimrath Gus se row äusserte sich bei derselben Gelegenheit über diese Frage folgendermaassen: Er habe gewiss stets dem abwartenden Verfahren in der Geburtshülfe gehuldigt, er verwerfe vor allen Dingen jeden überflüssigen operativen Eingriff auf das Strengste - dennoch könne er das von Dührssen vorgeschlagene Verfahren keineswegs so ohne weiteres verurtheilen. Jeder Geburtshelfer, dem eine einigermaassen grössere Erfahrung zur Seite steht, werde sich einer Reihe von Fällen *) erinnern, wenn dieselben allerdings immerhin selten genug seien, wo eine schleunige Entbindung im Interesse der Mutter und des Kindes dringend indicirt erschien, wo aber dieselbe entweder gar nicht oder nur mit Opferung des Kindes und schwerem Nachtheil für die Mutter ermöglicht werden konnte einzig und allein deshalb, weil der Muttermund und die mütterlichen Weichtheile die Entbindung nach unseren bisherigen Anschauungen unmöglich erscheinen liessen. Dass man in solchen peinlichen Fällen hier und da mit der manuellen Dilatation des Muttermundes zum Ziele kommen könne, ist auch jedem Geburtshelfer geläufig; wie äusserst selten diese Methode aber wirklich Erfolg habe, dürfte nicht minder bekannt sein. Es sei seiner Auffassung nach nun ein entschiedenes Verdienst Dührssen's, durch sein Verfahren gezeigt zu haben, wie man in derartigen schweren Fällen die Entbindung ausführen könne, und zwar, wie seine Fälle doch unzweifelhaft gezeigt haben, ohne Nachtheil für Mutter und Kind. Er begrüsse das Verfahren Dührssen's also als einen entschiedenen Fortschritt unserer geburtshülflichen Technik, möchte aber allerdings auf das Ernstlichste vor zu schneller Verallgemeinerung desselben warnen. Die näheren Indicationen für das Verfahren können erst festgestellt werden durch Prüfung seitens erfahrener und in der operativen Technik und der Antisepsis absolut geschulter Geburtshelfer; erst nach sorgfältiger Sichtung der so gemachten Erfahrungen kann die Methode Allgemeingut der Aerzte werden.

Auch Börner ${ }^{1}$ ) hat jüngst erklärt, die Kliniker hätten die Pficht, mein Verfahren unparteiisch zu prüfen. Bis jetzt ist dies nicht geschehen, und nur mit Bedauern kann ich von solchen

*) Anmerkung. Zu diesen Fällen gehört auch die Eklampsie, was Gusserow Löhlein gegenüber auf dem Bonner Gynäkologencongresse hervorhob, da Löhlein von neuen Operationen bei Eklampsie nur den Kaiserschnitt erwähnt hatte.

1) Sammlung klinischer Vortrăge. Neue Folge. Nr. 18. 
Fällen lesen, wie die von Börner, Levy ${ }^{1}$ ), Walich ${ }^{2}$ ) und Anderen publicirten, wo man, statt das Kind zu perforiren, durch meine Methode mit Sicherheit ein lebendes. Kind erhalten hätte. Für mich hat dieselbe auch weiterhin die Erwartungen erfüllt, die ich auf sie gesetzt. In allen Fällen habe ich keine Mutter und kein Kind verloren, habe dem Kinde gar keine und der Mutter in einzelnen Fällen kleine Nachtheile in Gestalt von nicht per primam geheilter Incisionen zugefügt - Nachtheile, die sich leicht repariren lassen, die auch nach vielen spontanen Geburten im Anschlusse an Zerreissungen der Weichtheile auftreten und die in Fällen, wo man durch meine Methode ein schwer bedrohtes mütterliches oder kindliches Leben rettet, absolut nicht ins Gewicht fallen können.

Man hat mir imputirt, dass ich bei jedem zweiten Geburtsfalle zu diesen Muttermundsincisionen schritte, man hat ferner an meinen Indicationen herumgemäkelt. Der erste Einwand ist hinfällig durch die im Verhältnisse $\mathrm{zu}$ einem grossen Material geringe Anzahl meiner Fälle, und was den zweiten anbetrifft, so habe ich niemals gerathen, wegen einfacher Wehenschwäche die Entbindung vorzunehmen, wie das z. B. in dem Schröder'schen Lehrbuche empfohlen wird. Meine Ansicht geht dahin, dass auf Grund einer solchen Empfehlung wegen blosser „Erschöpfung oder Aufregung" der Kreissenden in der Praxis viel zu häufig zur Zange gegriffen wird, dass also im Allgemeinen in der Geburtshülfe viel zu viel operirt wird. Weiter aber bin ich der Meinung, dass in gewissen Kategorien von Fällen fehlerhafter Weise nicht operirt oder eine falsche resp. conservativer Geburtshïlfe nicht entsprechende Operation gewählt wird. Ich verwerfe also speciell bei rigidem Muttermunde alter Erstgebärender, bei denen Gefahr für die Mutter oder das Kind auftritt, sowohl das einfache Abwarten als auch die Perforation und halte im Princip nur dasjenige Operationsverfahren für das richtige, welches eine völlige Erweiterung des Muttermundes durch das Messer erzielt und so die schonende Extraction eines lebenden Kindes ermöglicht.

Wenn ich also für Fälle, in welchen zwecks Erhaltung auch des kindlichen Lebens doch operirt werden muss, vielfach die Operationsmethode ändern will und die für die Mutter und das Kind ungefährlichste Operation empfehle, so weiss ich nicht, inwiefern ich einer geburtshülflichen Vielgeschäftigkeit Thür und Thor öffne.

1) Virchow-Hirsch'scher Jahresbericht 1889. Kapitel Geburtshülfe.

2) a. a. 0 . 
Diese geburtshülfliche Vielgeschäftigkeit ist in den Verhältnissen der Praxis begründet und Schuld der Aerzte, wie es Schuld der Gynäkologen ist, dass in der Gynäkologie viel zu viel operirt wird.

Damit komme ich zu einer andern Frage, welche mit dem anatomischen Ergebnisse der vorliegenden Arbeit innig zusammenhängt, nämlich zur Frage der Indication und Technik der Portioamputation. So segensreich wie diese Operation bei chronischer Metritis sein kann, indem sie mit eínem Schlage alle Beschwerden beseitigt, so treten doch in einer Reihe von Fällen statt der alten neue Beschwerden auf, welche an Intensität die alten gewöhnlich bedeutend übertreffen und deren Therapie eine viel schwierigere ist. Diese Beschwerden finden ihr Substrat in einer Para- resp. Perimetritis posterior, welche sich im Anschlusse und infolge der Operation entwickelt hatte. Selbstverständlich habe ich nicht grössere derartige Exsudate im Sinne - diese lassen sich durch die Anti- resp. Asepsis verhüten -, sondern empfindliche Narbenstränge im hinteren Scheidengewölbe, die ich in unserer gynäkologischen Poliklinik in einer ganzen Reihe von Fällen nach Portioamputation feststellen konnte, in Fällen, die von den verschiedensten Berliner Gynäkologen operirt worden waren. In den meisten dieser Fälle liess sich aus der Anamnese mit voller Sicherheit feststellen, dass die vorhandenen Beschwerden erst nach der Amputation aufgetreten waren. Während vorher nur Blutungen, geringe Kreuzschmerzen, Drängen nach unten vorhanden waren, stellten sich nach der Operation heftige Schmerzen im Kreuze und Leibe, Schmerzen bei der Defäcation und der Cohabitation ein. In diesen Fällen fand ich theils empfindliche Stränge im hinteren Scheidengewölbe, welche direct mit den vom Muttermunde ausstrahlenden Narbensträngen in Verbindung standen, theils höher hinauf eine Infiltration der Douglas'schen Falten und zwischen ihnen noch empfindliche Stränge, die ihrer Lage nach als perimetritische gedeutet werden mussten. In anderen Fällen strahlten Narbenzüge in das vordere Scheidengewölbe und die Parametrien aus, indessen war ihre Empfindlichkeit eine weit geringere. Diese unangenehmen Folgezustände der Operation sind nicht ganz selten. Denn auch Jacobi Müller ${ }^{1}$ ) fand sie unter 450 Fällen der Martin'schen Anstalt in 33 Fällen, in denen sie vor der Operation fehlten. Unter weiteren 74, mit Perimetritis complicirten Fällen bildete sich 24 Mal direct im Anschlusse an die Operation ein Recidiv aus, und

1) Zeitschrift für Geburtshülfe und Gynäkologie, Bd. XI 
eine ganze Anzahl von Recidiven schloss sich noch an die Herausnahme der Fäden an.

Die Para- bezw. Perimetritis posterior post amputationem portionis, die also selbst bei vollendetster Ausführung der Operation entstehen kann, stellt nun meiner Ansicht nach einen Folgezustand der Operation dar, welcher viel schlimmer ist, als das Uebel, zu dessen Bekämpfung man die Operation wählte. Die Hauptaufgabe der Technik dieser Operation muss daher darin be-

Fig. 11.

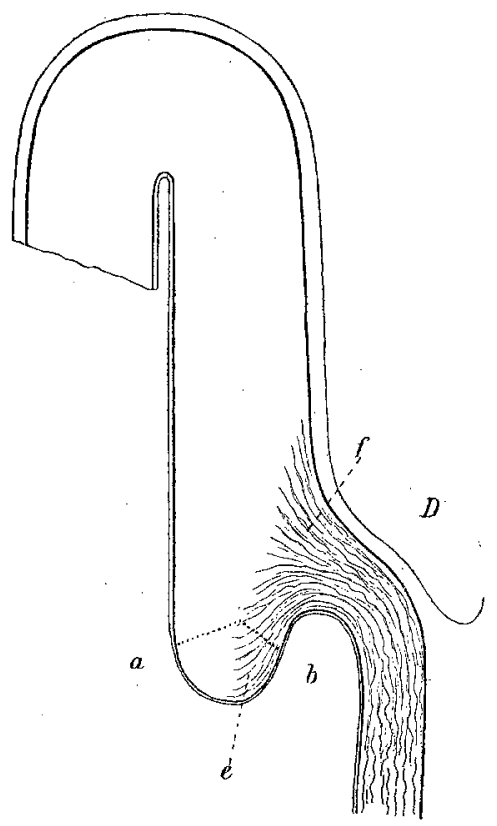

Umformung der Wunde $a b$ nach d. Excision. $c c,=$ im Douglas liegende Naht. $e=$ unteres retrahirtes Bündel. $f=$ oberes gedehntes Bündel. $D=$ Douglas'scher Raum.

stehen, diese Folgezustände mit Sicherheit zu vermeiden. Um dies zu können, müssen die Ursachen der entzündlichen Nachkrankheiten nach der Portioamputation klar gestellt werden. Sehr einfach erklären sich dieselben, wenn die Portio im Niveau des hinteren Scheidengewölbes abgeschnitten wird, denn dann liegen die Nähte in dem retrocervicalen Gewebe bezw. fassen das Peritoneum mit. Für die Fälle aber, wo man die Portio tiefer absetzte, scheinen mir die prädisponirenden Ursachen der Para- und Peri- 
metritis posterior in den Eingangs meiner Arbeit geschilderten anatomischen Verhältnissen begründet zu sein.

Sobald nämlich der Schnitt bei der Portioamputation die hintere Fläche der Portio trifft (Fig. 11) - und diese Schnittführung muss man wegen der Grösse der mitzuentfernenden Erosion meistens sogar in den Fällen wählen, wo man sich auf die Schröder'schen Excisio mucosae cervicis beschränkt -, so wird der periphere, an elastischen Fasern reiche Abschnitt der Portio von dem centralen Abschnitte abgetrennt und retrahirt sich mitsammt der Portioschleimhaut auf die hintere Vaginalwand. Hierdurch wird auch das obere Gewebsbündel, welches von der Scheide auf den Uterus ïbergeht und ebenfalls elastische Fasern enthält, stark gezerrt und somit verdünnt. Dicht hinter dieser äusserst dünnen Schicht liegt aber das retrocervicale Gewebe und das Peritoneum des Douglas'schen Raumes. Der Grund der Wunde wird also nur in der Nähe des Cervicalcanals von Uterussubstanz gebildet, nach hinten zu liegt die Wunde im Scheidengewölbe, ihr Grund wird von dem äusseren, noch dazu verdünnten Theile der Vaginalwand gebildet, dem das Peritoneum dicht anliegt (Fig. 12). Letzteres kann sogar eröffnet werden, wenn die hintere Lippe vom Cervicalcanale aus abgetragen und an dem theilweise losgelösten Stücke stark gezogen wird. Hierdurch zerrt man leicht das obere Bindegewebsbündel der Scheide und mit ihm das Peritoneum in die Schnittlinie hinein. Entgegen Hegar und Kaltenbach') glaube ich also, dass eine Eröffnung des Peritonealsackes vorkommen kann, ohne dass man die Durchtrennung zu hoch, innerhalb der invertirten Scheide, vorgenommen hat. Bei der Excision selbst wird dieses Ereigniss meistens gar nicht bemerkt, weil der eröffnete Peritonealzipfel sich sofort retrahirt. Durch die Umformung der Wunde an der hinteren Lippe wird es nun bedingt, dass bei der üblichen Methode der Vernähung, bei welcher im Cervicalcanale ein- und am hinteren Wundrande ausgestochen wird, die Nadel durch das retrocervicale Gewebe und häufig genug durch das Peritoneum hindurchgeführt wird. So wird also selbst bei unterhalb des Scheidenansatzes ausgeführter Amputation durch die Stichkanäle eine directe Communication zwischen retrouterinem Bindegewebe und Peritoneum einerseits und der Cervical- und Vaginalschleimhaut andererseits geschaffen. Bei der Schwierigkeit, diese Schleimbäute keimfrei zu machen resp. zu erhalten, ist also eine

1) Die operative Gynäkologie. 
Entzündung im Bereiche der Stichcanäle, zumal wenn man mit Seide näht, leicht möglich, und diese Entzündung ist dann eben eine lokalisirte Para- resp. Perimetritis.

Um dieses üble Ereigniss zu vermeiden, scheint es mir wichtig, die Wunde in der Tiefe durch versenkte Catgutnähte zu schliessen und Cervical- und Scheidenschleimhaut nur durch ganz oberflächliche Suturen mit einander $\mathbf{z u}$ vereinigen.

An der vorderen Lippe findet ganz dieselbe Retraction statt. Hier ist sie indessen von geringerer praktischer Bedeutung, da die entstehenden Narben wegen der grösseren Entfernung von der vorderen Peritonealtasche gar keine oder nur geringe Schmerzen erzeugen.

Mögen die beigebrachten Thatsachen nicht nur zu einer Modification der Technik auffordern, sondern auch dazu dienen, die Indication für die Portioamputation und die analogen Operationen, wie die Excisio mucosae cervicis und die kegelmantelförmige Excision nach Simon (Marckwald) etwas strenger zu formuliren! In der Mehrzahl der Fälle verschwinden die der Metritis chronica zugeschriebenen Beschwerden durch die Beseitigung der Endometritis vermittels Curettement resp. Aetzungen, durch die Schliessung eines Cervixrisses, durch die Beseitigung den Uterus dislocirender para- und perimetritischer Narbenstränge vermittels Massagebehandlung nach Thure Brandt. Gegen Stenosen des Uterus besitzen wir wirksam therapeutische Methoden in der Discision mit nachfolgender Umsäumung nach $\mathrm{Heg}$ a $\mathrm{r}$ und in der Dilatation. Die letztere lässt sich am raschesten und schmerzlosesten durch die Apostoli'sche Methode erzielen.

Mit diesen Anschauungen, welche auch diejenigen meines hochverehrten Chefs sind, bekennen wir uns zu den Grundsätzen conservativer Chirurgie, die ja auch auf den übrigen Gebieten der Gynäkologie immer mehr Anhänger gewinnen.

Vielleicht bietet die bei der Portioamputation stattfindende Retraction auch eine Erklärung für die Vorliebe, mit welcher das Cancroid der Portio auf die Scheide und das paracervicale und paravaginale Gewebe übergeht - eine Thatsache, welche von C. Ruge und J. Veit') gefunden wurde. Aehnlich wie der Schnitt trennt auch die bösartige Wucherung den mit der Vagina zusammenhängenden peripheren Theil der

1) Zeitschrift für Geburtshülfe und Gynäkologie, Bd. VII. 
Portio von dem centralen ab. Dies geschieht natürlich nur allmälig, so dass zur Zeit nur kleine Faserpartien sich auf die Vagina hin retrahiren. Die Carcinompartikel aber, die sie mit sich ziehen, genügen, um die krebsige Wucherung auf die Umgebung des Uterus zu übertragen, und zwar schon auf die tieferen Schichten der Vaginalwand, bei noch auf die Portioschleimhant um den Muttermund herum beschränktem Cancroid.

Noch eine praktische Bemerkung. - Sie betrifft die der Totalexstirpation vorausgeschickte Excision der carcinomatösen Wucherungen. Macht man die Excision im Gesunden, so kann der Schnitt in der Tiefe auf eingesprengte Krebsnester treffen, welche sich dann mit dem gesunden, noch retractionsfähigen Gewebe so weit auf die Vaginalwand hin retrahiren, dass sie bei der nachfolgenden Totalexstirpation zurïckgelassen werden und schnell ein Recidiv erzeugen. Es dürfte sich daher empfehlen, die Excision hinderlicher carcinomatöser Wucherungen vor der Totalexstirpation principiell stets innerhalb der Geschwulst selbst vorzunehmen, weil in dem Carcinom selbst keine Retraction mehr stattfindet.

An dem ersten oben beschriebenen Präparate (Taf. XI u. XII, Fig. 2 u. 3) habe ich das Verhalten der elastischen Fasern zu der vorhandenen Erosion genau geschildert. Es ist dies deswegen geschehen, weil ich glaube, dass die Beziehungen der elastischen Fasern zu den Erosionen einen Anhaltspunkt für die vielumstrittene Entstehung der Erosionen abzugeben im Stande sind. Die Erosion unseres Präparates ist in Heilung begriffen. Man sieht, wie Hofmeier ') das beschrieben hat, dass eine mächtige Schicht Plattenepithel sich über eine Reihe von Drüsen (7 an der Zahl) hinzieht, von denen ein Theil vollständig abgerundet, also wohl schon völlig abgeschnürt ist. Unter dem Plattenepithel liegen elastische Fasern, welche sich genau so weit erstrecken, als das Plattenepithel reicht. Wir sehen ferner die sechs äusseren Drüsen der Erosion von einem Netze elastischer Fasern umsponnen, welches mit der unter dem Plattenepithel gelegenen Schicht elastischer Fasern in innigem Zusammenhange steht. Woher kommt es nun, dass diese sechs äusseren Drüsen von elastischen Fasern umsponnen werden, die innerste (Nr. 7 auf Fig. 3) aber nicht? Es rührt dies meiner Ansicht nach daher, dass die sechs äusseren, auf dem Schnitte sichtbaren Drüsen durch Einsenkungen von Zellen des Rete Malpighi

1) Zeitschrift für Geburtshülfe, Bd. IV, S. 349. 
entstanden sind, in der Weise, wie das von C. Ruge und J. Veit') beschrieben worden ist.

Als Ausgangspunkt einer näheren Betrachtung über die Entstehung der unter dem Plattenepithele gelegenen Drüsen unseres Falles stelle ich zunächst die Thatsache hin, dass sich normaliter unter dem Rete Malpighi des Plattenepithels eine zusammenhängende Schicht elastischer Fasern findet, die an der Cervicalschleimhaut fehlt. Ich habe diese Beobachtung übereinstimmend an allen meinen Präparaten, an Uteri von Neugeborenen, an einem Uterus einer Nullipara (s. S. 19), an Uteri von Mehrgebärenden und an zwei puerperalen Uteri gemacht. Entstehen nun die Drüsen der Erosion, so wie Ruge und Veit es beschreiben, aus Einsenkungen der Zellen des Rete Malpighi, so müssen diese Einsenkungen die Schicht elastischer Fasern, welche unter dem Rete gelegen ist, einstülpen, so muss sich um die fertigen Drüsen eine Schicht elastischer Fasern herumziehen, welche mit der unter dem Rete gelegenen Schicht elastischer Fasern continuirlich zusammenhängt. Sind die Drüsen der Erosion dagegen Drüsen der Cervicalschleimhaut, so dürfen sie keıne sie umspinnende Schicht elastischer Fasern aufzuweisen haben. In unserem Präparate sehen wir nun, dass bis auf die innerste die übrigen Drüsen von elastischen Fasern umgeben sind. Diese Drüsen sind also durch Umformung und Einstülpung der Zellen des Rete Malpighi im Sinne von Ruge und Veit entstanden. Die innere Drüse gehört zur Cervicalschleimhaut. Ihre Mündung liegt jedenfalls in der Cervicalschleimhaut. Wir haben es hier nur mit einer Ausbuchtung einer Cervicaldrüse in die tieferen Schichten der Portioschleimhaut hinein zu thun.

Gegen die Deutung des Befundes liesse sich vielleicht der Einwand erheben, dass Hand in Hand mit den Heilungsvorgängen der Erosion, wie sie in dem geschilderten Präparate zu beobachten sind, auch eine Neubildung elastischer Fasern geht, die dann secundär auch um die Drüsen herumwachsen. Dieser Einwand wird durch folgende weitere Präparate hinfällig:

1. Curettement und Excision einer Erosion der vorderen Lippe bei einer 37 jährigen $V$ para. Die mikroskopische Untersuchung ergiebt, dass die elastischen Fasern nur bis zur ersten Drüse (Fig. 13, bis a)

1) Zeitschrift für Geburtshülfe, Bd. II, S. 434. 
reichen, während der Plattenepithelsaum noch eine kleine Strecke weiter, über drei Drüsenlumina hinweg zieht (bis $b$ ). Mit der Verdünnung und dem Schwinden dieses Saumes geht Hand

Fig. 13. in Hand eine nach der Cervicalschleimhaut immer mehr

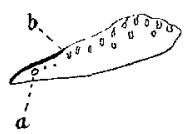
zunehmende kleinzellige Infiltration der unter dem Plattenepithel gelegenen Gewebsschichten. Im Bereiche der Erosion ist diese infiltrirte Gewebsschicht nur an einzelnen Stellen von Cylinderepithel bedeckt, grösstentheils liegt sie frei zu Tage.

Fig. 13 stellt den Schnitt, grob umrissen, in natürlicher Grösse dar.

2. 46jährige VII para, Prolaps der vorderen Vaginalwand, Hypertrophie der Portio, linksseitiger, innen über das Scheidengewölbe hinausreichender Cervixriss. Kolporrhaphia anterior et posterior und Excision der freiliegenden Cervicalschleimhant mit dem angrenzenden Theil der Portioschleimhaut; die Sagittalschnitte durch beide Lippen weisen gar keine elastischen Fasern auf. Der Plattenepithelsaum zieht

Fig. 14.

Fig. 15.
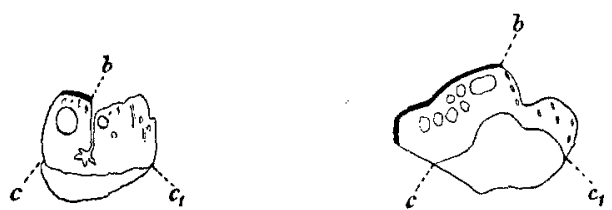

über eine Reihe von cystisch erweiterten Drüsen hinweg bis $b$ (Fig. 14 vordere, Fig. 15 hintere Lippe). Schon makroskopisch setzt sich die Schleimhaut bei $c c$, deutlich gegen das unterliegende Muskelgewebe ab. Person.

3. Die bereits Seite 19 beschriebene Erosion einer 72jährigen

4. 30jährige Person, die $1 \mathrm{Mal}$ geboren. Ektropium, Cervixriss. Excisio mucosae cervicis, Emmet. Das excidirte Gewebe (Fig. 16) der vorderen Lippe weist auf Frontalschnitten einen kurzen Saum Plattenepithel auf, unter welchem ein Flechtwerk elastischer Fasern gelegen ist. Es folgt dann ein reines Granulationsgewebe ohne eine Spur von Cervicaldrüsen. Zwischen $a$ und $a$, liegt ein Saum elastischer Fasern, welche die Ausläufer der elastischen Fasern aus dem

Fig. 16.

Fig. 17.
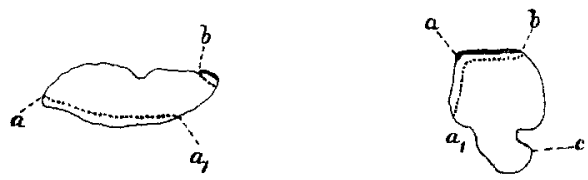

nicht mit excidirten peripheren Abschnitte der Lippe darstellen. Auf Sagittalschnitten durch die hintere Lippe reicht das Plattenepithel bis $b$ (Fig. 17), anter demselben liegt ebenfalls ein Flechtwerk elastischer 
Fasern, und die Ausläufer der elastischen Fasern des tiefen Netzes nehmen den Bezirk $a a$, ein. Bei $e$ finden sich Reste von Cervicaldrüsen.

5. 32 jährige Fran, die vor fünf Jahren zuletzt geboren. Amputation beider Lippen, wobei aber von der Vorder- bezw. Rückseite der

Fig. 18.

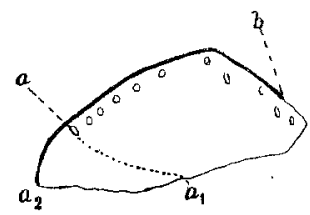

Fig. 19.

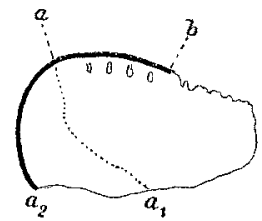

Portio nur wenig weggenommen wird. Auf den Sagittalschnitten durch beide Lippen (Fig. 18 u. 19) nehmen die elastischen Fasern den Bezirk $a a_{2} a_{1}$ ein; an der vorderen Lippe erreichen die unter dem Plattenepithel oberfläehlich gelegenen Fasern die erste Drüse, in der hinteren Lippe bleiben sie noch ein kleines Stäck von der ersten entfernt. Das Plattenepithel überzieht das excidirte Stück der vorderen Lippe fast vollständig.

6. Excision einer Erosion beider Lippen bei einer 34 jährigen IV para. An der vorderen Lippe (Fig. 20) nehmen die elastischen

Fig. 20.

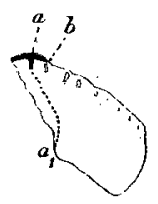

Fig. 21.

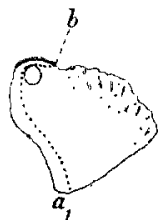

Fasern den von der Linie $a$ a, abgegrenzten kleinen Zipfel ein. Besonders interessant ist das Verhalten der oberflächlichen, unter dem Plattenepithel gelegenen elastischen Fasern, die, bei $a$ endigend, eine daselbst gelegene, solide Einstïlpung des Plattenepithels umspinnen. Das Plattenepithel reicht noch etwas weiter, bis $b$. An der hinteren Lippe reichen elastische Fasern und Plattenepithel bis $b$, ohne dass aber die elastischen Fasern die Innenseite der daselbst gelegenen abgeschnürten Drüse umspinnen (Fig. 21).

An diesem letzten Präparat sieht man also direct die Entstehung einer Erosionsdrüse im Sinne von Ruge und Veit. Wir haben eine solide Einstülpung des Plattenepithels vor uns, welche yon elastischen Fasern umsponnen wird. Indessen ist diese Bildung auf eine einzige periphere Drüse beschränkt. Das, was als Erosion sichtbar war und excidirt wurde, war bis auf diese eine 
Drüse ein wahres Ectropium, eine Ausstülpung der Cervicalschleimhaut, da die übrigen Drüsen nicht von elastischen Fasern umgeben sind.

An den fünf anderen Präparaten sehen wir, dass an der ersten, am meisten peripher gelegenen Drüse die elastischen Fasern endigen, und zwar hören sie entweder mit dem Plattenepithel zusammen auf, oder das Plattenepithel geht noch eine Strecke weiter über eine Reihe von Drüsen hinweg. Diese theilweise cystisch entarteten Drüsen (Ovula Nabothi) stammen, da ihnen umkreisende elastische Fasern fehlen, von der Cervicalschleimhaut ab. Es handelt sich also auch in diesen fünf Fällen um ein Ektropium. Wie erklärt sich nun der theilweise Ueberzug des Ektropium mit Plattenepithel? Ich bin geneigt mit Doléris 1 ) anzunehmen, dass es sich hier um eine primäre Ulceration ${ }^{2}$ ) der Cervicalschleimhaut handelte, die dann secundär vom Plattenepithel übernarbt wurde. Eine solche Ulceration sehen wir in dem vierten Präparat. Die elastischen Fasern folgen aber dem Plattenepithel nicht auf das Gebiet des Ektropium, sie respectiren auch bei pathologischen Processen ihre physiologische Grenze, die den Beginn der Cervicalschleimhaut bezeichnet. Gewiss stellt diese Uebernarbung eines Ektropium, welche durch die Anwendung von Holzessig und Chlorzink befördert wird, keine ideale Heilung dar - darin stimme ich mit Doléris und $\operatorname{Stratz}^{3}$ ) vollständig überein —, denn die gespannten Follikel bleiben in der Tiefe liegen; allein ich glaube, dass man deswegen nicht immer die Excisio mucosae cervicis zu machen braucht, sondern dass man meistens mit einem ausgiebigen Curettement der gesammten Uterusschleimhaut auskommt, an welche sich dann, wie schon erwähnt, die Emmet'sche Operation anzuschliessen hat.

Wenn ich noch meine Meinung über das Verhältniss zwischen Erosion und Ektropium äussern darf, so nehme ich im Gegensatze zu Doléris ${ }^{4}$ ) an, dass eine Erosion im Sinne von C. Ruge und J. Veit existirt, dass sie aber bei Frauen, die geboren haben, seltener ist, als das Ektropium, und dass sie meistens eine räumlich beschränkte Bildung darstellt, dass also in der Regel der grössere Antheil einer Erosion im Sinne von Ruge und Veit von einem wahren Ektropium gebildet wird.

1) vgl. die verschiedenen Artikel in Nouvelles archives d'obstétrique et de gyn. 1889 und 1890.

2) Siehe auch Döderlein, Centralblatt für Gynäkologie 1889, S. 99.

3) Zeitschrift für Geburtshülfe, Bd. XVIII.

4) a. a. 0 . 
In der vorliegenden Arbeit habe ich eine anätomische $\mathrm{Zu}$ sammensetzung der Portio vaginalis beschrieben, wie sie meines Wissens noch nicht beschrieben worden ist. Bei Gelegenheit des internationalen Congresses zu Berlin machte mich Doléris darauf aufmerksam, dass Forget ${ }^{1}$ ) bereits eine analoge Beschreibung gegeben habe. Ich muss dem widersprechen. Forget beschreibt nämlich (S. 52) folgende Zusammensetzung der Vagina aus fünf Schichten, von denen die ersten vier auf das Collum übergehen:

1) Die Śchleimhaut.

2) Eine submucöse Schicht, aus dichtem Bindegewebe bestehend - couche fibreuse.

3) Ein spongiöses, erectiles Gewebe, welches elastische Fasern zu enthalten scheint.

4) Eine Schicht glatter Muskulatur.

5) Eine lockere Bindegewebsschicht, die dem Peritoneum des Douglas'schen Raumes anliegt.

Die fibröse Schicht hört nach Forget vorn 5 oder $6 \mathrm{~mm}$ und hinten 9 oder $10 \mathrm{~mm}$ über dem äusseren Muttermunde auf, die erectile unter 3 genante Schicht verbreitert sich und bildet so einen Kegel, dessen Basis sich dem Collum direct anlegt und mit dessen Substanzverschmilat (s'identifie), indem sie sich aber hauptsächlich an seiner Oberfläche auszubreiten scheint.

Die Muskelschicht geht in die oberflächlichen, longitudinalen Muskelfasern des Uterus über.

An einer anderen Stelle (S. 198) sagt Forget noch Folgendes: „Am Collum bilden die Muskelfasern einige schwache circuläre Ringe, unter welchen nach dem Orificium externum hin als Complement ein Gewebe existirt, das noch nicht genau bestimmt ist und dessen Histologie noch geschrieben werden muss. Es ist dasjenige, welches ich als eine Ausbreitung des erectilen Gewebes der 'Scheide betrachte."

Forget hat also das Richtige geahnt, indessen die Ausbreitung des elastischen Gewebes in der Portio nur an der Oberfläche angenommen, während nach meiner Anschauung das Netzwerk

1) Etude pratique et philosophique du col de la matrice. Paris 1849, Labé.

Nachdem Doléris sich vergeblich bemüht, mir dieses Werk zu verschaffen, habe ich es in dem 150. Katalog der Moser'schen Buchhandlung in Tübingen (ausgegeben: April 1891) gefunden und von dort bezogen. 
elastischer Fasern normaliter die ganze äussere Hälfte der Portio vaginalis einnimmt.

Mit einer genaueren Beschreibung der elastischen Fasern des ganzen Uterus und der Portio beschäftigt sich dann meines Wissens nur noch die schöne Arbeit von Acconci. ${ }^{1}$ ) Auch diese Arbeit kam mir erst zu Gesicht, nachdem ich meine Beobachtungen zum Theil schon (August 1890) publicirt hatte. Acconci nimmt nicht die zwei von mir beschriebenen Schichten der Portio an, obgleich man an der von ihm gegebenen Abbildung (Fig. 1) deutlich sieht, dass die äussere Schicht der Portio viel reicher an elastischen Fasern ist. Er betont aber die Wichtigkeit dieser reichen Faserentwicklung für die Erweiterung des Muttermundes bei der Geburt und bezieht, ebenso wie ich, die Rigidität des Collum auf eine mangelhafte Entwickelung der elastischen Fasern, ohne freilich für diese Behauptung irgendwelche Beweise beizubringen.

\section{Schlussfolgerungen.}

1) Die ausgebildete Portio besteht aus zwei gleich grossen Abschnitten, einem peripheren und einem centralen, der Cervicalschleimhaut angrenzenden Abschnitte.

2) Der periphere unterscheidet sich anatomisch von dem centralen durch das Vorwiegen des Bindegewebes über die Muskulatur und den Reichthum an elastischen Fasern.

3) Die elastischen Fasern bilden ein oberflächliches, unter dem Plattenepithel gelegenes Netzwerk, welches bis zur Cervicalschleimhaut reicht, und ein tiefes gröberes Netzwerk, welches die Gefässe umspinnt. Beide Netzwerke hängen untereinander und mit den elastischen Fasern der Scheide zusammen.

4) Diese anatomische Anordnung ermöglicht bei der Geburt die Erweiterung des Muttermundes durch eine vollständige Auseinanderfaltung der Portio. Hierbei verbleibt der centrale $\mathrm{Ab}-$ schnitt der Portio dem Uterus, der periphere dagegen verschmilzt völlig mit der Vaginalwand.

5) Diese Auseinanderfaltung der Portio kommt durch das harmonische Zusammenwirken zweier Factoren zu Stande: Durch

1) Contributo allo Studio dell' anatomia e fisiologia dell' utero gestante e partoriente. Estratto dal giornale della regia academia di Medicina 1890, Nr. 7. 
den Zug der Uterusmuskulatur von oben her und durch den horizontal wirkenden Druck der Fruchtblase (bei gesprungener Blase) des vorliegenden Theiles.

6) Bei Neugeborenen fehlt das tiefe Netz elastischer Fasern, ebenso bei alten Frauen; bei Frauen mit infantilem Habitus, die erst spät concipiren, ist es mangelhaft entwickelt.

7) Bei alten Erstgebärenden liegt die Rigidität der Portio und die Schwierigkeit der Erweiterung des Muttermundes an einem Mangel an elastischen Fasern (im Bereich des tiefen Netzes). Derselbe beruht entweder auf einer mangelhaften Entwickelung (bei Frauen mit infantilem Habitus) oder auf einem Altersschwund der elastischen Fasern.

8) In diesen Fällen sind mechanische Dehnungsversuche irrationell. Bei Gefahr für Mutter oder Kind entbinde man mit Hülfe von tiefen Incisionen.

9) Infolge des Gehaltes der Portio an elastischen Fasern kommt es bei der Portioamputation zu eigenthümlichen Umformungen der gesetzten Wundflächen, welche an der hinteren Lippe die Entstehung einer Para- bezw. Perimetritis posterior begünstigen.

10) Die Ausbreitung des Portiocancroids auf das paracervicale Gewebe hängt möglicherweise auch mit dem Gehalt des peripheren Abschnittes der Portio an elastischen Fasern zusammen.

11) Da die elastischen Fasern in der Umgebung der Cervicaldrüsen fehlen, unter dem Plattenepithel aber vorhanden sind, so ermöglicht dieses Verhalten in jedem Falle eine Entscheidung darüber, ob die Drüsen einer Erosion der Cervicalschleimhaut angehören (Ektropion) oder aus dem Plattenepithel entstanden sind (Erosion im Sinne von Ruge und Veit).

Frklärung der Abbildungen auf Tafel XI u. XII.

Fig. 1.

Sagittalschnitt durch die vordere Lippe einer Mehrgebärenden.

$\left.\begin{array}{l}a=\text { Plattenepithel der Portio. } \\ b=\text { oberflächliches Netz elastischer Fasern. } \\ c=\text { tiefes Netz elastischer Fasern. }\end{array}\right\} \begin{gathered}\text { Peripherer Abschnitt der } \\ \text { Portio. }\end{gathered}$

$\left.\begin{array}{l}d=\text { glatte Muskulatur. } \\ e=\text { Cervicalschleimhaut. }\end{array}\right\}$ Centraler Abschnitt der Portio.

Fig. 2.

Sagittalschnitt durch die vordere Lippe einer Mehrgebărenden. $a=$ Plattenepithel einer heilenden Erosion der Portio. 
294 Dührssen, Beitrag zur Anatomie der Portio vaginalis uteri.

$\dot{b}=$ oberflächliches Netz elastischer Fasern, welches auch die Drüsen $1,2,3$ und 4 umspinnt.

$\ddot{e} d=$ Erosionsdrüsen.

$d=$ glatte Muskulatur.

Fig. 3.

Sagittalschnitt durch die vordere Lippe einer Mehrgebärenden (Fortsetzung von Fig. 2).

$a=$ Plattenepithèl einer heilenden Erosion der Portio.

$b=$ oberflächliches Netz elastischer Fasern, welches die Drüse 4 und 5 umspinnt, an der Drüse 7 ganz fehlt, an den Drüsen 6 und 8 am Fundus der Drüsen ebenfalls fehlt.

$e d=$ Erosionsdrüsen.

$d=$ glatte Muskulatur.

Fig. 5 (schwache Vergrösserung).

Sagittalschnitt durch die vordere Lippe and Vaginalwand eines puerperalen Uterus.

$a=$ centraler Abschnitt der Portio.

$b=$ peripherer Abschnitt der Portio mit elastischen Fasern.

$c=$ Partie, welche Fig. 6 bei starker Vergrösserung zeigt.

$v=$ Vaginalwand.

sch $=$ Scheidengewölbe.

Fig. 6.

Partie von Fig. 5 bei starker Vergrösserung (Zeiss $D D$, Compensationsocular 8).

Zwischen den auseinandergezerrten Bindegewebsbündeln liegen die blau gefärbten elastischen Fasern.

Fig. 8.

Sagittalschnitt durch die vordere Lippe eines neugeborenen Mädchens. $a=$ Plattenepithel.

$b=$ peripherer Abschnitt der Portio mit elastischen Fasern.

$d$ und $e=$ centraler Abschnitt der Portio ohne elastische Fasern; $d=$ Muskulatur, $e=$ Cervicalschleimhaut. 